\title{
Hybrid method for equilibrium problems and variational inclusions
}

\author{
Shahram Rezapour ${ }^{1,2,3^{*}}$ (D) and Seyyed Hasan Zakeri ${ }^{4}$
}

\author{
"Correspondence: \\ shahramrezapour@duytan.edu.vn; \\ sh.rezapour@mail.cmuh.org.tw; \\ sh.rezapour@azaruniv.ac.ir; \\ rezapourshahram@yahoo.ca \\ ${ }^{1}$ Institute of Research and \\ Development, Duy Tan University, \\ Da Nang, 550000, Vietnam \\ ${ }^{2}$ Faculty of Natural Sciences, Duy \\ Tan University, Da Nang, 550000, \\ Vietnam \\ Full list of author information is \\ available at the end of the article
}

\begin{abstract}
By providing a new iterative method our aim is finding a common element of the set of fixed points of two nonexpansive mappings, the set of solutions to a variational inclusion and the set of solutions of a generalized equilibrium problem in a real Hilbert space. We review the strong convergence of the new iterative method in the framework of Hilbert spaces. Finally, we show that our main result is a generalization for some known theorems in this field.
\end{abstract}

MSC: Primary 46N10; secondary 47N10

Keywords: Maximal monotone operator; Nonexpansive map; Variational inclusion; Generalized equilibrium problem; Inverse strongly monotone map

\section{Introduction}

Let $H$ be a real Hilbert space, let $C$ be a nonempty closed convex subset of $H$, let $A: C \rightarrow H$ be a nonlinear map, and let $F$ be a bifunction from $C \times C$ to $\mathbb{R}$. In 2008, Takahashi et al. [1] considered the generalized equilibrium problem: Finding $x \in C$ such that

$$
F(x, y)+\langle A x, y-x\rangle \geq 0 \quad(\forall y \in C) .
$$

The set of solutions of (1) is denoted by $\operatorname{GEP}(F, A)$. If $A=0$, then problem (1) becomes the equilibrium problem: Finding $x \in C$ such that

$$
F(x, y) \geq 0 \quad(\forall y \in C) .
$$

Problem (2) was studied by Blum et al. [2] in 1994. The set of solutions of (2) is denoted by $\operatorname{EP}(F)$. If $F(x, y)=0$ for all $x, y \in C$, then problem (1) becomes the variational inequality problem: Finding $x \in C$ such that

$$
\langle A x, y-x\rangle \geq 0 \quad(\forall y \in C)
$$

Problem (3) was studied by Hartman et al. [3] in 1966 and has been extensively in the literature (see, e.g., [4-10]). The set of solutions of (3) is denoted by $\operatorname{VI}(C, A)$. If $C=H$, then $\operatorname{VI}(H, A)=A^{-1}(0)=\{x \in H: A x=0\}$. Recall that a mapping $A: C \rightarrow H$ is said to be

(c) The Author(s) 2020. This article is licensed under a Creative Commons Attribution 4.0 International License, which permits use, sharing, adaptation, distribution and reproduction in any medium or format, as long as you give appropriate credit to the original author(s) and the source, provide a link to the Creative Commons licence, and indicate if changes were made. The images or other third party material in this article are included in the article's Creative Commons licence, unless indicated otherwise in a credit line to the material. If material is not included in the article's Creative Commons licence and your intended use is not permitted by statutory regulation or exceeds the permitted use, you will need to obtain permission directly from the copyright holder. To view a copy of this licence, visit http://creativecommons.org/licenses/by/4.0/. 
monotone if $\langle A u-A v, u-v\rangle \geq 0$ for all $u, v \in C[6,7,11]$. A mapping $A$ is said to be $\alpha$ strongly monotone if there exists a positive real number $\alpha$ such that $\langle A u-A v, u-v\rangle \geq$ $\alpha\|u-v\|^{2}$ for all $u, v \in C[6,7,11]$. A mapping $A$ is said to be $\alpha$-inverse strongly monotone if there exists a positive real number $\alpha$ such that $\langle A u-A v, u-v\rangle \geq \alpha\|A u-A v\|^{2}$ for all $u, v \in C[6,7,11]$. In such a case, $A$ is said to be $\alpha$-inverse-strongly monotone.

Let $T: C \rightarrow C$ be a mapping. We denote by $F(T)$ the fixed-point set of $T$, that is, $F(T)=$ $\{x \in C: T(x)=x\}$. A mapping $T$ is said to be $L$-Lipschitz if there exists $L \geq 0$ such that $\|T u-T v\| \leq L\|u-v\|$ for all $u, v \in C$. The mapping $T$ is called nonexpansive if $L=1$. It is also called contraction if $L<1$. Note that any $\alpha$-inverse strongly monotone mapping $A$ is Lipschitz and $\|A u-A v\| \leq \frac{1}{\alpha}\|u-v\|[6,7,11]$. There are a lot works associated with the fixed point algorithms for nonexpansive mappings (see, e.g., [12-23]).

Let $A: H \rightarrow H$ be a single-valued nonlinear map, and let $B: H \rightarrow 2^{H}$ be a set-valued mapping. The variational inclusion is finding $p \in H$ such that

$$
\theta \in A(p)+B(p)
$$

where $\theta$ is the zero vector in $H$. For $A=0$, (4) becomes the inclusion problem introduced by Rockafellar [24]. The effective domain of $B$ is denoted by $D(B)$, that is, $D(B)=\{x \in$ $H: B x \neq \emptyset\}$. The graph of $B$ is $G(B)=\{(u, v) \in H \times H: v \in B u\}$. A set-valued mapping $B$ is said to be monotone if $\langle x-y, f-h\rangle \geq 0$ for all $x, y \in D(B), f \in B x$, and $h \in B y$ [25]. A monotone operator $B$ is maximal if the graph $G(B)$ of $B$ is not properly contained in the graph of any other monotone mapping [25]. Also, a monotone mapping $B$ is maximal if and only if $\langle x-y, f-h\rangle \geq 0((x, f) \in H \times H(y, h) \in G(B))$ implies $f \in B x$ [25]. For a maximal monotone operator $B$ on $H$ and $r>0$, we define the single-valued operator $J_{r}^{B} x=$ $(I+r B)^{-1}: H \rightarrow D(B)$, which is called the resolvent of $B$ for $r$. It is well known that $J_{r}^{B} x$ is firmly nonexpansive, that is, $\left\langle x-y, J_{r}^{B} x-J_{r}^{B} y\right\rangle \geq\left\|J_{r}^{B} x-J_{r}^{B} y\right\|^{2}$ for all $x, y \in H$ (see [13]), and that a solution of (4) is a fixed point of $J_{r}^{B}(I-r A)$ for all $r>0$ [25].

A basic problem for maximal monotone operator $B$ is finding

$$
x \in H \quad \text { such that } \quad 0 \in B x .
$$

A known method for solving problem (5) is the proximal point algorithm: $x_{1}=x \in H$, and

$$
x_{n+1}=J_{r_{n}}^{B} x_{n} \quad(n \geq 1)
$$

where $J_{r_{n}}^{B}=\left(I+r_{n} B\right)^{-1}$ and $\left\{r_{n}\right\} \subset(0, \infty)$. Then Rockafellar [24, 26] proved that the sequence $\left\{x_{n}\right\}$ converges weakly to an element of $B^{-1}(0)$ (see also [27]). In the literature, there are a large number references associated with the proximal point algorithm [2729]. In 2011, Shehu [8] suggested the following iterative sequence. Let $\left\{x_{n}\right\}$ be the sequence generated by

$$
\left\{\begin{array}{l}
x_{1} \in C, \\
F\left(y_{n}, y\right)+\left\langle A x_{n}, y-y_{n}\right\rangle+\frac{1}{r_{n}}\left\langle y-y_{n}, y_{n}-x_{n}\right\rangle \geq 0 \quad(\forall y \in C), \\
x_{n+1}=a_{n} x_{n}+\left(1-a_{n}\right) T\left[\beta_{n} f\left(x_{n}\right)+\left(1-\beta_{n}\right) J_{r_{n}}^{B}\left(y_{n}-r_{n} A y_{n}\right)\right] \quad(\forall n \geq 1) .
\end{array}\right.
$$

Under appropriate conditions, the author proved that the sequence $\left\{x_{n}\right\}$ converges strongly to a point $P_{F(T) \cap(A+B)^{-1}(0) \cap \mathrm{GEP}(F, A)} u$ [8]. Our goal in this paper is to present an 
iterative method that converges strongly to a common element of the fixed point set of two nonexpansive mappings and the zero set of the sums of maximal monotone operators in Hilbert spaces. Our results extend and improve some related old results.

\section{Preliminaries}

Let $H$ be a real Hilbert space, and let $C$ be a nonempty closed convex subset of $H$. It is well known that, for any $x \in H$, there exists a unique nearest point in $C$, denoted by $P_{C}(x)$, such that $\left\|x-P_{C}(x)\right\|=\inf _{y \in C}\|x-y\|=: d(x, C)$. It is well known that $P_{C}$ is nonexpansive monotone mapping from $H$ onto $C,\left\langle x-P_{C} x, z-P_{C} x\right\rangle \leq 0,\|x-z\|^{2} \geq\left\|x-P_{C} x\right\|^{2}+\left\|z-P_{C} x\right\|^{2}$ for all $x \in H$ and $z \in C$, and $\left\langle P_{C} x-P_{C} z, x-z\right\rangle \geq\left\|P_{C} x-P_{C} z\right\|^{2}$ for all $z, x \in H$ (see [13]). Let $A$ be a monotone mapping from $C$ into $H$. In the context of the variational inequality problem, it is easy to see that from the relation $\left\langle x-P_{C} x, z-P_{C} x\right\rangle \leq 0$ we have

$$
p \in \operatorname{VI}(C, A) \Leftrightarrow p=P_{C}(p-\lambda A p) \text { for some } \lambda>0 .
$$

For solving the equilibrium problem for a a bifunction $F: C \times C \rightarrow \mathbb{R}$, we assume that $F$ satisfy the following conditions:

$\left(A_{1}\right) F(x, x)=0$ for all $x \in C$,

$\left(A_{2}\right) F$ is monotone, that is, $F(x, y)+F(y, x) \leq 0$ for all $x, y \in C$,

$\left(A_{3}\right)$ for each $x, y, z \in C, \lim _{t \rightarrow 0} F(t z+(1-t) x, y) \leq F(x, y)$,

$\left(A_{4}\right)$ for each $x \in C$, the function $y \mapsto F(x, y)$ is convex and lower semicontinuous.

Put $F(x, y)=\langle A x, y-x\rangle$ for $x, y \in C$. Then we see that the equilibrium problem (2) is reduced to the variational inequality (3). We need the following results.

Lemma 2.1 ([2,30]) Let $C$ be a nonempty closed convex subset of $H$, and let $F$ be a bifunction from $C \times C$ to $\mathbb{R}$ satisfying $\left(A_{1}\right)-\left(A_{4}\right)$. For $r>0$ and $x \in H$, consider the map $T_{r}: H \rightarrow C$ defined by $T_{r}(x)=\left\{z \in C: F(z, y)+\frac{1}{r}\langle y-z, z-x\rangle \geq 0\right.$ for all $\left.y \in C\right\}$. Then $T_{r}(x) \neq \emptyset$ for all $x \in H, T_{r}$ is single-valued, $\mathrm{EP}(F)$ is closed and convex, $F\left(T_{r}\right)=\mathrm{EP}(F)$, and $T_{r}$ is firmly nonexpansive, that is, $\left\|T_{r}(x)-T_{r}(y)\right\|^{2} \leq\left\langle T_{r}(x)-T_{r}(y), x-y\right\rangle$ for all $x, y \in H$.

Lemma 2.2 ([31]) Let $C$ be a nonempty closed convex subset of $H$, and let $F$ be a bifunction from $C \times C$ to $\mathbb{R}$ satisfying $\left(A_{1}\right)-\left(A_{4}\right)$. Define the multivalued mapping $A_{F}$ from $H$ into itself by $A_{F} x=\{z \in C: F(z, y) \leq\langle y-x, z\rangle$ for all $y \in C\}$ whenever $x \in C$ and $A_{F} x=\emptyset$ otherwise. Then $A_{F}$ is a maximal monotone operator with the domain $T_{r}(x)=\left(I+r A_{F}\right)^{-1} x$ for all $x \in H$ and $r>0$.

Lemma 2.3 ([32]) Let $H$ be a real Hilbert space, let $C$ be a closed convex subset of $H$, and let $T: C \rightarrow C$ be a nonexpansive mapping. Then $(I-T)$ is demiclosed at zero, that is, if $\left\{x_{n}\right\}$ is a sequence in $C$ such that $x_{n} \rightarrow x$ and $T x_{n}-x_{n} \rightarrow 0$, then $x=T(x)$.

Lemma 2.4 ([33]) Let $\left\{x_{n}\right\}$ be a sequence of nonnegative real numbers satisfying

$$
x_{n+1} \leq\left(1-\lambda_{n}\right) x_{n}+\gamma_{n}
$$

where $\left\{\lambda_{n}\right\}$ is a sequence in $(0,1)$, and $\gamma_{n}$ is a sequence with $\sum_{n=0}^{\infty} \lambda_{n}=\infty$ and $\limsup _{n \rightarrow \infty} \gamma_{n} \leq 0$ or $\sum_{n=0}^{\infty}\left|\gamma_{n} \lambda_{n}\right|<\infty$. Then $\lim _{n \rightarrow \infty} x_{n}=0$. 
Lemma 2.5 ([34]) Let $H$ be a real Hilbert space, let $x_{j} \in H$, and let $a_{j} \in[0,1], j=1,2,3$, be such that $a_{1}+a_{2}+a_{3}=1$. Then we have

$$
\left\|a_{1} x_{1}+a_{2} x_{2} z+a_{3} x_{3}\right\|^{2}=a_{1}\left\|x_{1}\right\|^{2}+a_{2}\left\|x_{2}\right\|^{2}+a_{3}\left\|x_{3}\right\|^{2}-\sum_{1 \leq i, j \leq 3} a_{i} a_{j}\left\|x_{i}-x_{j}\right\|^{2} .
$$

Lemma 2.6 ([31]) Let B be a maximal monotone operator on $H$. Then we have

$$
\frac{\lambda-r}{r}\left\langle J_{\lambda}^{B} x-J_{r}^{B} x, J_{\lambda}^{B} x-x\right\rangle \geq\left\|J_{\lambda}^{B} x-J_{r}^{B} x\right\|^{2} \quad(\forall \lambda, r>0 \text { and } x \in H) .
$$

\section{Main results}

Now we are ready to state and prove our main results.

Theorem 3.1 Let $C$ be a nonempty closed convex subset of $H$, let $F$ be a bifunction from $C \times C$ to $\mathbb{R}$ satisfying $\left(A_{1}\right)-\left(A_{4}\right)$, let $A$ be an $\alpha$-inverse strongly monotone mapping from $C$ into $H$, let $M$ be a $\beta$-inverse strongly monotone map from $C$ into $H$, and let $B$ be a maximal monotone operator on $H$ with domain contained in $C$. Assume that $S, T: C \rightarrow C$ are two nonexpansive mappings such that $\Omega=F(T) \cap F(S) \cap(M+B)^{-1}(0) \cap \operatorname{GEP}(F, A) \neq \emptyset$ and $f: C \rightarrow C$ is a contraction map with the constant $\rho \in(0,1)$. Suppose that $\left\{b_{n}\right\},\left\{a_{n}\right\}$, and $\left\{\mu_{n}\right\}$ are some sequences in $(0,1)$ and $\left\{x_{n}\right\},\left\{y_{n}\right\}$, and $\left\{z_{n}\right\}$ are the sequences generated by

$$
\left\{\begin{array}{l}
x_{1} \in C, \\
F\left(y_{n}, y\right)+\left\langle A x_{n}, y-y_{n}\right\rangle+\frac{1}{r_{n}}\left\langle y-y_{n}, y_{n}-x_{n}\right\rangle \geq 0 \quad(\forall y \in C), \\
z_{n}=\mu_{n} x_{n}+\left(1-\mu_{n}\right) J_{\lambda_{n}}^{B}\left(y_{n}-\lambda_{n} M y_{n}\right), \\
x_{n+1}=P_{C}\left[b_{n} f\left(x_{n}\right)+\left(1-b_{n}\right)\left(a_{n} S z_{n}+\left(1-a_{n}\right) T y_{n}\right)\right] \quad(\forall n \geq 1) .
\end{array}\right.
$$

Suppose the following conditions hold:

$$
\begin{aligned}
& \left(d_{1}\right) 0<c \leq \lambda_{n} \leq d<2 \beta, \lim _{n \rightarrow \infty}\left|\lambda_{n}-\lambda_{n-1}\right|=0, \\
& \left(d_{2}\right) 0<a \leq r_{n} \leq b<2 \alpha, \lim _{n \rightarrow \infty}\left|r_{n}-r_{n-1}\right|=0, \\
& \left(d_{3}\right) \lim _{n \rightarrow \infty} b_{n}=0, \sum_{n=1}^{\infty} b_{n}=\infty, \sum_{n=1}^{\infty}\left|b_{n}-b_{n-1}\right|<\infty, \\
& \left(d_{4}\right) \sum_{n=1}^{\infty}\left|\mu_{n}-\mu_{n-1}\right|<\infty, \sum_{n=1}^{\infty}\left|a_{n}-a_{n-1}\right|<\infty .
\end{aligned}
$$

Then $\left\{x_{n}\right\}$ converges strongly to a point $q \in \Omega$, which is the unique solution to the variational inequality $\langle(I-f) q, x-q\rangle \geq 0$ for all $x \in \Omega$.

Proof First, we show that $I-\lambda_{n} M$ is nonexpansive. Let $x, y \in C$ and $0<\lambda_{n}<2 \beta$. Then

$$
\begin{aligned}
\left\|\left(I-\lambda_{n} M\right) x-\left(I-\lambda_{n} M\right) y\right\|^{2} & =\left\|(x-y)-\lambda_{n}(M x-M y)\right\|^{2} \\
& \leq\|x-y\|^{2}-2 \lambda_{n}\langle x-y, M x-M y\rangle+\lambda_{n}^{2}\|M x-M y\|^{2} \\
& \leq\|x-y\|^{2}-\lambda_{n} \beta\|M x-M y\|^{2}+\lambda_{n}^{2}\|M x-M y\|^{2} \\
& =\|x-y\|^{2}+\lambda_{n}\left(\lambda_{n}-2 \beta\right)\|M x-M y\|^{2} \\
& \leq\|x-y\|^{2} .
\end{aligned}
$$

Thus $I-\lambda_{n} M$ is nonexpansive. Note that $y_{n}$ can be rewritten as $y_{n}=T_{r_{n}}\left(x_{n}-r_{n} A x_{n}\right)$ for $n \geq 1$. Let $q \in \Omega$. From $\left(d_{2}\right)$ and Lemma 2.1 we have

$$
\left\|y_{n}-q\right\|^{2}=\left\|T_{r_{n}}\left(x_{n}-r_{n} A x_{n}\right)-q\right\|^{2}
$$




$$
\begin{aligned}
& \left.=\| T_{r_{n}}\left(x_{n}-r_{n} A x_{n}\right)-T_{r_{n}}\left(q-r_{n} A q\right)\right) \|^{2} \\
& \leq\left\|\left(x_{n}-r_{n} A x_{n}\right)-\left(q-r_{n} A q\right)\right\|^{2} \\
& =\left\|x_{n}-q\right\|^{2}+r_{n}\left(r_{n}-2 \alpha\right)\left\|A x_{n}-A q\right\|^{2} \leq\left\|x_{n}-q\right\|^{2} .
\end{aligned}
$$

By (6) and (7), since $J_{\lambda_{n}}^{B}$ is nonexpansive, we have

$$
\begin{aligned}
\left\|z_{n}-q\right\|^{2} & =\left\|\mu_{n} x_{n}+\left(1-\mu_{n}\right) J_{\lambda_{n}}^{B}\left(y_{n}-\lambda_{n} M y_{n}\right)-q\right\|^{2} \\
& =\left\|\mu_{n}\left(x_{n}-q\right)+\left(1-\mu_{n}\right)\left(J_{\lambda_{n}}^{B}\left(y_{n}-\lambda_{n} M y_{n}\right)-q\right)\right\|^{2} \\
& \leq \mu_{n}\left\|x_{n}-q\right\|^{2}+\left(1-\mu_{n}\right)\left\|J_{\lambda_{n}}^{B}\left(y_{n}-\lambda_{n} M y_{n}\right)-q\right\|^{2} \\
& \leq \mu_{n}\left\|x_{n}-q\right\|^{2}+\left(1-\mu_{n}\right)\left\|J_{\lambda_{n}}^{B}\left(y_{n}-\lambda_{n} M y_{n}\right)-J_{\lambda_{n}}^{B}\left(q-\lambda_{n} M q\right)\right\|^{2} \\
& \leq \mu_{n}\left\|x_{n}-q\right\|^{2}+\left(1-\mu_{n}\right)\left\|\left(y_{n}-\lambda_{n} M y_{n}\right)-\left(q-\lambda_{n} M q\right)\right\|^{2} \\
& \leq \mu_{n}\left\|x_{n}-q\right\|^{2}+\left(1-\mu_{n}\right)\left(\left\|y_{n}-q\right\|^{2}+\lambda_{n}\left(\lambda_{n}-2 \beta\right)\left\|M y_{n}-M q\right\|^{2}\right) \\
& \leq\left\|x_{n}-q\right\|^{2}+\left(1-\mu_{n}\right) \lambda_{n}\left(\lambda_{n}-2 \beta\right)\left\|M y_{n}-M q\right\|^{2} \leq\left\|x_{n}-q\right\|^{2} .
\end{aligned}
$$

Hence

$$
\begin{aligned}
\left\|x_{n+1}-q\right\| & =\left\|P_{C}\left[b_{n} f\left(x_{n}\right)+\left(1-b_{n}\right)\left(a_{n} S z_{n}+\left(1-a_{n}\right) T y_{n}\right)\right]-P_{C}(q)\right\| \\
& \leq\left\|\left[b_{n} f\left(x_{n}\right)+\left(1-b_{n}\right)\left(a_{n} S z_{n}+\left(1-a_{n}\right) T y_{n}\right)\right]-q\right\| \\
& \leq b_{n}\left\|f\left(x_{n}\right)-q\right\|+\left(1-b_{n}\right)\left[a_{n}\left\|S z_{n}-q\right\|+\left(1-a_{n}\right)\left\|T y_{n}-q\right\|\right] \\
& \leq b_{n}\left\|f\left(x_{n}\right)-q\right\|+\left(1-b_{n}\right)\left[a_{n}\left\|x_{n}-q\right\|+\left(1-a_{n}\right)\left\|y_{n}-q\right\|\right] \\
& \leq b_{n}\left\|f\left(x_{n}\right)-q\right\|+\left(1-b_{n}\right)\left\|x_{n}-q\right\| \\
& \leq b_{n}\left(\left\|f\left(x_{n}\right)-f(q)\right\|+\|f(q)-q\|\right)+\left(1-b_{n}\right)\left\|x_{n}-q\right\| \\
& \leq b_{n}\left(\rho\left\|x_{n}-q\right\|+\|f(q)-q\|\right)+\left(1-b_{n}\right)\left\|x_{n}-q\right\| \\
& \leq\left(1-b_{n}(1-\rho)\right)\left\|x_{n}-q\right\|+b_{n}\|f(q)-q\| \\
& \leq \max \left\{\left\|x_{n}-q\right\|, \frac{\|f(q)-q\|}{(1-\rho)}\right\} \\
& \vdots \\
& \leq \max \left\{\left\|x_{1}-q\right\|, \frac{\|f(q)-q\|}{(1-\rho)}\right\} .
\end{aligned}
$$

Thus $\left\{x_{n}\right\}$ is bounded, and the sequences $\left\{y_{n}\right\}$ and $\left\{z_{n}\right\}$ are bounded as well. From $y_{n}=$ $T_{r_{n}}\left(x_{n}-r_{n} A x_{n}\right)$ and $y_{n-1}=T_{r_{n-1}}\left(x_{n-1}-r_{n-1} A x_{n-1}\right)$ we obtain

$$
F\left(y_{n}, y\right)+\left\langle A x_{n}, y-y_{n}\right\rangle+\frac{1}{r_{n}}\left\langle y-y_{n}, y_{n}-x_{n}\right\rangle \geq 0 \quad(\forall y \in C)
$$

and

$$
F\left(y_{n-1}, y\right)+\left\langle A x_{n-1}, y-y_{n-1}\right\rangle+\frac{1}{r_{n-1}}\left\langle y-y_{n-1}, y_{n-1}-x_{n-1}\right\rangle \geq 0 \quad(\forall y \in C) .
$$


By substituting $y=y_{n-1}$ into (10) and $y=y_{n}$ into (11), we find

$$
F\left(y_{n}, y_{n-1}\right)+\left\langle A x_{n}, y_{n-1}-y_{n}\right\rangle+\frac{1}{r_{n}}\left\langle y_{n-1}-y_{n}, y_{n}-x_{n}\right\rangle \geq 0
$$

and

$$
F\left(y_{n-1}, y_{n}\right)+\left\langle A x_{n-1}, y_{n}-y_{n-1}\right\rangle+\frac{1}{r_{n-1}}\left\langle y_{n}-y_{n-1}, y_{n-1}-x_{n-1}\right\rangle \geq 0 .
$$

Now from $\left(A_{2}\right)$ we get $\left\langle A x_{n-1}-A x_{n}, y_{n}-y_{n-1}\right\rangle+\left\langle y_{n-1}-y_{n}, \frac{y_{n}-x_{n}}{r_{n}}-\frac{y_{n-1}-x_{n-1}}{r_{n-1}}\right\rangle \geq 0$, and so

$$
\begin{aligned}
0 \leq & \left\langle y_{n}-y_{n-1}, r_{n}\left(A x_{n-1}-A x_{n}\right)+\frac{r_{n}}{r_{n-1}}\left(y_{n-1}-x_{n-1}\right)-\left(y_{n}-x_{n}\right)\right\rangle \\
= & \left\langle y_{n-1}-y_{n}, y_{n}-y_{n-1}+\left(1-\frac{r_{n}}{r_{n-1}}\right) y_{n-1}+\left(x_{n-1}-r_{n-1} A x_{n-1}\right)\right\rangle \\
& -\left\langle y_{n+1}-y_{n},\left(x_{n}-r_{n} A x_{n}\right)+x_{n-1}-\frac{r_{n}}{r_{n-1}} x_{n-1}\right\rangle \\
= & \left\langle y_{n-1}-y_{n}, y_{n}-y_{n-1}+\left(1-\frac{r_{n}}{r_{n-1}}\right)\left(y_{n-1}-x_{n-1}\right)\right. \\
& \left.+\left(x_{n-1}-r_{n-1} A x_{n-1}\right)-\left(x_{n}-r_{n} A x_{n}\right)\right\rangle .
\end{aligned}
$$

This implies that $\left\|y_{n}-y_{n-1}\right\|^{2} \leq\left\|y_{n}-y_{n-1}\right\|\left[\left|1-\frac{r_{n}}{r_{n-1}}\right|\left\|y_{n-1}-x_{n-1}\right\|+\left\|x_{n}-x_{n-1}\right\|\right]$, and so

$$
\left\|y_{n}-y_{n-1}\right\| \leq \frac{\left|r_{n}-r_{n-1}\right|}{r_{n-1}}\left\|y_{n-1}-x_{n-1}\right\|+\left\|x_{n}-x_{n-1}\right\|
$$

Set $w_{n}=J_{\lambda_{n}}^{B}\left(y_{n}-\lambda_{n} M y_{n}\right)$ and $u_{n}=y_{n}-\lambda_{n} M y_{n}$ for $n \geq 1$. By using Lemma 2.6 we obtain

$$
\begin{aligned}
\left\|w_{n}-w_{n-1}\right\|= & \left\|J_{\lambda_{n}}^{B}\left(y_{n}-\lambda_{n} M y_{n}\right)-J_{\lambda_{n-1}}^{B}\left(y_{n-1}-\lambda_{n-1} M y_{n-1}\right)\right\| \\
= & \left\|J_{\lambda_{n}}^{B} u_{n}-J_{\lambda_{n-1}}^{B} u_{n-1}+J_{\lambda_{n}}^{B} u_{n-1}-J_{\lambda_{n}}^{B} u_{n-1}\right\| \\
\leq & \left\|\left(y_{n}-\lambda_{n} M y_{n}\right)-\left(y_{n-1}-\lambda_{n-1} M y_{n-1}\right)\right\| \\
& +\left\|J_{\lambda_{n}}^{B} u_{n-1}-J_{\lambda_{n-1}}^{B} u_{n-1}\right\| \\
\leq & \left\|\left(y_{n}-\lambda_{n} M y_{n}\right)-\left(y_{n-1}-\lambda_{n} M y_{n-1}\right)+\left(\lambda_{n-1}-\lambda_{n}\right) M y_{n-1}\right\| \\
& +\frac{\left|\lambda_{n-1}-\lambda_{n}\right|}{\lambda_{n-1}}\left\|J_{\lambda_{n-1}}^{B} u_{n-1}-u_{n-1}\right\| \\
\leq & \left\|y_{n}-y_{n-1}\right\|+\left|\lambda_{n-1}-\lambda_{n}\right|\left\|M y_{n-1}\right\|+\frac{\left|\lambda_{n-1}-\lambda_{n}\right|}{\lambda_{n-1}}\left\|J_{\lambda_{n-1}}^{B} u_{n-1}-u_{n-1}\right\| \\
\leq & \left\|x_{n}-x_{n-1}\right\|+\frac{\left|r_{n}-r_{n+1}\right|}{r_{n+1}}\left\|y_{n+1}-x_{n+1}\right\|+\left|\lambda_{n-1}-\lambda_{n}\right|\left\|M y_{n-1}\right\| \\
& +\frac{\left|\lambda_{n-1}-\lambda_{n}\right|}{\lambda_{n-1}}\left\|J_{\lambda_{n-1}}^{B} u_{n-1}-u_{n-1}\right\|,
\end{aligned}
$$

which gives

$$
\left\|z_{n}-z_{n-1}\right\|=\left\|\left[\mu_{n} x_{n}+\left(1-\mu_{n}\right) w_{n}\right]-\left[\mu_{n-1} x_{n-1}+\left(1-\mu_{n-1}\right) w_{n-1}\right]\right\|
$$




$$
\begin{aligned}
= & \left\|\mu_{n}\left(x_{n}-x_{n-1}\right)+\left(1-\mu_{n}\right)\left(w_{n}-w_{n-1}\right)+\left(\mu_{n}-\mu_{n-1}\right)\left(x_{n-1}-w_{n-1}\right)\right\| \\
\leq & \mu_{n}\left\|x_{n}-x_{n-1}\right\|+\left|\mu_{n}-\mu_{n-1}\right|\left\|x_{n-1}-w_{n-1}\right\|+\left(1-\mu_{n}\right)\left\|w_{n}-w_{n-1}\right\| \\
\leq & \left\|x_{n}-x_{n-1}\right\|+\left|\mu_{n}-\mu_{n-1}\right|\left\|x_{n-1}-w_{n-1}\right\| \\
& +\left(1-\mu_{n}\right)\left(\left|\lambda_{n-1}-\lambda_{n}\right|\left\|M y_{n-1}\right\|+\frac{\left|r_{n}-r_{n+1}\right|}{r_{n+1}}\left\|y_{n+1}-x_{n+1}\right\|\right. \\
& \left.+\frac{\left|\lambda_{n-1}-\lambda_{n}\right|}{\lambda_{n-1}}\left\|J_{\lambda_{n-1}}^{B} u_{n-1}-u_{n-1}\right\|\right) .
\end{aligned}
$$

Set $t_{n}=a_{n} S z_{n}+\left(1-a_{n}\right) T y_{n}$ for $n \geq 1$. By using (12) and last inequality we have

$$
\begin{aligned}
\left\|t_{n}-t_{n-1}\right\|= & \| a_{n}\left(S z_{n}-S z_{n-1}\right)+\left(1-a_{n}\right)\left(T y_{n}-T y_{n-1}\right) \\
& +\left(a_{n}-a_{n-1}\right)\left(S z_{n-1}-T y_{n-1}\right) \| \\
\leq & a_{n}\left\|S z_{n}-S z_{n-1}\right\|+\left(1-a_{n}\right)\left\|T y_{n}-T y_{n-1}\right\| \\
& +\left|a_{n}-a_{n-1}\right|\left\|S z_{n-1}-T y_{n-1}\right\| \\
\leq & a_{n}\left\|z_{n}-z_{n-1}\right\|+\left(1-a_{n}\right)\left\|y_{n}-y_{n-1}\right\|+\left|a_{n}-a_{n-1}\right|\left\|S z_{n-1}-T y_{n-1}\right\| \\
\leq & \left\|x_{n}-x_{n-1}\right\|+\frac{\left|r_{n}-r_{n+1}\right|}{r_{n+1}}\left\|y_{n+1}-x_{n+1}\right\|+\left|a_{n}-a_{n-1}\right|\left\|S z_{n-1}-T y_{n-1}\right\| \\
& +\left(1-\mu_{n}\right) a_{n}\left(\left|\lambda_{n-1}-\lambda_{n}\right|\left\|M y_{n-1}\right\|+\frac{\left|\lambda_{n-1}-\lambda_{n}\right|}{\lambda_{n-1}}\left\|J_{\lambda_{n-1}}^{B} u_{n-1}-u_{n-1}\right\|\right) \\
& +a_{n}\left|\mu_{n}-\mu_{n-1}\right|\left\|x_{n-1}-w_{n-1}\right\|,
\end{aligned}
$$

which implies that

$$
\begin{aligned}
\left\|x_{n+1}-x_{n}\right\|= & \left\|P_{C}\left[b_{n} f\left(x_{n}\right)+\left(1-b_{n}\right) t_{n}\right]-P_{C}\left[b_{n-1} f\left(x_{n-1}\right)+\left(1-b_{n-1}\right) t_{n-1}\right]\right\| \\
\leq & \left\|\left[b_{n} f\left(x_{n}\right)+\left(1-b_{n}\right) t_{n}\right]-\left[b_{n-1} f\left(x_{n-1}\right)+\left(1-b_{n-1}\right) t_{n-1}\right]\right\| \\
\leq & \left\|b_{n}\left(f\left(x_{n}\right)-f\left(x_{n-1}\right)\right)+\left(1-b_{n}\right)\left(t_{n}-t_{n-1}\right)\right\| \\
& +\left\|\left(b_{n}-b_{n-1}\right)\left(f\left(x_{n-1}\right)-t_{n-1}\right)\right\| \\
\leq & b_{n}|| f\left(x_{n}\right)-f\left(x_{n-1}\right)\left\|+\left|b_{n}-b_{n-1}\right|\right\| f\left(x_{n-1}\right)-t_{n-1} \| \\
& +\left(1-b_{n}\right)\left\|t_{n}-t_{n-1}\right\| \\
\leq & b_{n} \rho\left\|x_{n}-x_{n-1}\right\|+\left|b_{n}-b_{n-1}\right|\left\|f\left(x_{n-1}\right)-t_{n-1}\right\|+\left(1-b_{n}\right)\left\|t_{n}-t_{n-1}\right\| \\
\leq & \left(1-b_{n}(1-\rho)\right)\left\|x_{n}-x_{n-1}\right\|+\left|b_{n}-b_{n-1}\right|\left\|f\left(x_{n-1}\right)-t_{n-1}\right\| \\
& +\frac{\left|r_{n}-r_{n+1}\right|}{a}\left\|y_{n+1}-x_{n+1}\right\|+\left|\mu_{n}-\mu_{n-1}\right|\left\|x_{n-1}-w_{n-1}\right\| \\
& +\left|\lambda_{n-1}-\lambda_{n}\right|\left\|M y_{n-1}\right\|+\frac{\left|\lambda_{n-1}-\lambda_{n}\right|}{c}\left\|J_{\lambda_{n-1}}^{B} u_{n-1}-u_{n-1}\right\| \\
& +\left|a_{n}-a_{n-1}\right|\left\|S z_{n-1}-T y_{n-1}\right\| \\
\leq & \left(1-b_{n}(1-\rho)\right)\left\|x_{n}-x_{n-1}\right\|+L\left[\left|b_{n}-b_{n-1}\right|+\left|\mu_{n}-\mu_{n-1}\right|\right. \\
& \left.+\frac{\left|r_{n}-r_{n+1}\right|}{a}+\left(1+\frac{1}{c}\right)\left|\lambda_{n-1}-\lambda_{n}\right|+\left|a_{n}-a_{n-1}\right|\right] \\
& +1 \mid
\end{aligned}
$$


where $L$ is a constant such that

$$
\begin{aligned}
L \geq & \max \left\{\sup _{n \geq 1}\left\|S z_{n-1}-T y_{n-1}\right\|, \sup _{n \geq 1}\left\|M y_{n}\right\|, \sup _{n \geq 1}\left\|J_{\lambda_{n-1}}^{B} u_{n-1}-u_{n-1}\right\|,\right. \\
& \left.\sup _{n \geq 1}\left\|f\left(x_{n-1}\right)-t_{n-1}\right\|, \sup _{n \geq 1}\left\|y_{n+1}-x_{n+1}\right\|, \sup _{n \geq 1}\left\|x_{n-1}-w_{n-1}\right\|\right\} .
\end{aligned}
$$

Now by using $\left(d_{1}\right),\left(d_{2}\right),\left(d_{3}\right),\left(d_{4}\right)$, and Lemma 2.4 we get

$$
\limsup _{n \rightarrow \infty}\left\|x_{n+1}-x_{n}\right\|=0
$$

From (6) we have

$$
\begin{aligned}
\left\|x_{n}-t_{n}\right\| & \leq\left\|x_{n}-x_{n+1}\right\|+\left\|x_{n+1}-t_{n}\right\| \\
& =\left\|x_{n}-x_{n+1}\right\|+\left\|p_{C}\left[b_{n} f\left(x_{n}\right)+\left(1-b_{n}\right) t_{n}\right]-P_{C}\left(t_{n}\right)\right\| \\
& \leq\left\|x_{n}-x_{n+1}\right\|+\left\|\left[b_{n} f\left(x_{n}\right)+\left(1-b_{n}\right) t_{n}\right]-t_{n}\right\| \\
& \leq\left\|x_{n}-x_{n+1}\right\|+b_{n}\left\|f\left(x_{n}\right)-t_{n}\right\| .
\end{aligned}
$$

Consequently, from the condition $\left(d_{3}\right)$ we obtain

$$
\lim _{n \rightarrow \infty}\left\|x_{n}-t_{n}\right\|=0
$$

Let $q=P_{\Omega} f(q)$. In addition, from (6), (8), Lemma 2.5, and (9) we get

$$
\begin{aligned}
\left\|x_{n+1}-q\right\|^{2}= & \left\|P_{C}\left[b_{n} f\left(x_{n}\right)+\left(1-b_{n}\right)\left(a_{n} S z_{n}+\left(1-a_{n}\right) T y_{n}\right)\right]-P_{C}(q)\right\|^{2} \\
\leq & \left\|\left[b_{n} f\left(x_{n}\right)+\left(1-b_{n}\right)\left(a_{n} S z_{n}+\left(1-a_{n}\right) T y_{n}\right)\right]-q\right\|^{2} \\
\leq & b_{n}\left\|f\left(x_{n}\right)-q\right\|^{2}+\left(1-b_{n}\right)\left[a_{n}\left\|S z_{n}-q\right\|^{2}+\left(1-a_{n}\right)\left\|T y_{n}-q\right\|^{2}\right. \\
& \left.-a_{n}\left(1-a_{n}\right)\left\|S z_{n}-T y_{n}\right\|\right] \\
\leq & b_{n}\left\|f\left(x_{n}\right)-q\right\|^{2}+\left(1-b_{n}\right)\left[a_{n}\left\|z_{n}-q\right\|^{2}+\left(1-a_{n}\right)\left\|y_{n}-q\right\|^{2}\right. \\
& \left.-a_{n}\left(1-a_{n}\right)\left\|S z_{n}-T y_{n}\right\|\right] \\
\leq & b_{n}\left\|f\left(x_{n}\right)-q\right\|^{2}+\left(1-b_{n}\right)\left(a_{n}\left\|x_{n}-q\right\|^{2}-a_{n}\left(1-a_{n}\right)\left\|S z_{n}-T y_{n}\right\|\right) \\
& +\left(1-b_{n}\right) a_{n}\left(1-\mu_{n}\right) \lambda_{n}\left(\lambda_{n}-2 \beta\right)\left\|M y_{n}-M q\right\|^{2} \\
& +\left(1-b_{n}\right)\left(1-a_{n}\right)\left(\left\|x_{n}-q\right\|^{2}+r_{n}\left(r_{n}-2 \alpha\right)\left\|A x_{n}-A q\right\|^{2}\right) \\
\leq & b_{n}\left\|f\left(x_{n}\right)-q\right\|+\left(1-b_{n}\right)\left(\left\|x_{n}-q\right\|-a_{n}\left(1-a_{n}\right)\left\|S z_{n}-T y_{n}\right\|\right) \\
& +\left(1-b_{n}\right) a_{n}\left(1-\mu_{n}\right) \lambda_{n}\left(\lambda_{n}-2 \beta\right)\left\|M y_{n}-M q\right\|^{2} \\
& +\left(1-b_{n}\right)\left(1-a_{n}\right) r_{n}\left(r_{n}-2 \alpha\right)\left\|A x_{n}-A q\right\|^{2},
\end{aligned}
$$

which yields

$$
\left(1-b_{n}\right)\left(1-a_{n}\right) r_{n}\left(2 \alpha-r_{n}\right) \cdot\left\|A x_{n}-A q\right\|^{2}
$$




$$
\begin{aligned}
\leq & b_{n}\left\|f\left(x_{n}\right)-q\right\|^{2}+\left\|x_{n}-q\right\|^{2}-\left\|x_{n+1}-q\right\|^{2} \\
& +\left(1-b_{n}\right) a_{n}\left(1-\mu_{n}\right) \lambda_{n}\left(\lambda_{n}-2 \beta\right)\left\|M y_{n}-M q\right\|^{2} \\
& -\left(1-b_{n}\right) a_{n}\left(1-a_{n}\right)\left\|S z_{n}-T y_{n}\right\|, \\
\left(1-b_{n}\right) a_{n}\left(1-\mu_{n}\right) \lambda_{n}\left(2 \beta-\lambda_{n}\right) \cdot\left\|M y_{n}-M q\right\|^{2} & \\
\leq & b_{n}\left\|f\left(x_{n}\right)-q\right\|^{2}+\left\|x_{n}-q\right\|^{2}-\left\|x_{n+1}-q\right\|^{2} \\
& +\left(1-b_{n}\right)\left(1-a_{n}\right) r_{n}\left(r_{n}-2 \alpha\right)\left\|A x_{n}-A q\right\|^{2} \\
& -\left(1-b_{n}\right) a_{n}\left(1-a_{n}\right)\left\|S z_{n}-T y_{n}\right\|,
\end{aligned}
$$

and

$$
\begin{aligned}
\left(1-b_{n}\right) a_{n}\left(1-a_{n}\right)\left\|S z_{n}-T y_{n}\right\| \leq & b_{n}\left\|f\left(x_{n}\right)-q\right\|^{2}+\left\|x_{n}-q\right\|^{2}-\left\|x_{n+1}-q\right\|^{2} \\
& +\left(1-b_{n}\right)\left(1-a_{n}\right) r_{n}\left(r_{n}-2 \alpha\right)\left\|A x_{n}-A q\right\|^{2} \\
& +\left(1-b_{n}\right) a_{n}\left(1-\mu_{n}\right) \lambda_{n}\left(\lambda_{n}-2 \beta\right)\left\|M y_{n}-M q\right\|^{2} .
\end{aligned}
$$

By using $\left(d_{1}\right),\left(d_{2}\right),\left(d_{3}\right)$, and (13) we get

$$
\lim _{n \rightarrow \infty}\left\|M y_{n}-M q\right\|=\lim _{n \rightarrow \infty}\left\|A x_{n}-A q\right\|=\lim _{n \rightarrow \infty}\left\|S z_{n}-T y_{n}\right\|=0 .
$$

Since $\left\|t_{n}-T y_{n}\right\| \leq a_{n}\left\|S z_{n}-T y_{n}\right\|$ and $\left\|t_{n}-S z_{n}\right\| \leq\left(1-a_{n}\right)\left\|T y_{n}-S z_{n}\right\|$, we obtain

$$
\lim _{n \rightarrow \infty}\left\|t_{n}-T y_{n}\right\|=\lim _{n \rightarrow \infty}\left\|t_{n}-S z_{n}\right\|=0 .
$$

Note that

$$
\begin{aligned}
\left\|w_{n}-q\right\|^{2}= & \left\|J_{\lambda_{n}}^{B}\left(y_{n}-\lambda_{n} M y_{n}\right)-J_{\lambda_{n}}^{B}\left(q-\lambda_{n} M q\right)\right\|^{2} \\
\leq & \left\langle\left(y_{n}-\lambda_{n} M y_{n}\right)-\left(q-\lambda_{n} M q\right), w_{n}-q\right) \\
= & \frac{1}{2}\left\|\left(y_{n}-\lambda_{n} M y_{n}\right)-\left(q-\lambda_{n} M q\right)\right\|^{2}+\frac{1}{2}\left\|w_{n}-q\right\|^{2} \\
& -\frac{1}{2}\left\|\left(y_{n}-\lambda_{n} M y_{n}\right)-\left(q-\lambda_{n} M q\right)-\left(w_{n}-q\right)\right\|^{2} \\
\leq & \frac{1}{2}\left[\left\|y_{n}-q\right\|^{2}+\left\|w_{n}-q\right\|^{2}-\left\|\left(y_{n}-w_{n}\right)-\lambda_{n}\left(M y_{n}-M q\right)\right\|^{2}\right] \\
= & \frac{1}{2}\left[\left\|y_{n}-q\right\|^{2}+\left\|w_{n}-q\right\|^{2}-\left\|y_{n}-w_{n}\right\|^{2}+2 \lambda_{n}\left\langle y_{n}-w_{n}, M y_{n}-M q\right\rangle\right. \\
& \left.-\lambda_{n}^{2}\left\|M y_{n}-M q\right\|^{2}\right],
\end{aligned}
$$

and so

$$
\left\|w_{n}-q\right\|^{2} \leq\left\|y_{n}-q\right\|^{2}-\left\|y_{n}-w_{n}\right\|^{2}+2 \lambda_{n}\left\|y_{n}-w_{n}\right\|\left\|M y_{n}-M q\right\| .
$$

By using Lemma 2.1 and (6) we have

$$
\left\|y_{n}-q\right\|^{2}=\left\|T_{r_{n}}\left(x_{n}-r_{n} A x_{n}\right)-T_{r_{n}}\left(q-r_{n} A q\right)\right\|^{2}
$$




$$
\begin{aligned}
\leq & \left\langle\left(x_{n}-r_{n} A x_{n}\right)-\left(q-r_{n} A q\right), y_{n}-q\right\rangle \\
= & \frac{1}{2}\left\|\left(x_{n}-r_{n} A x_{n}\right)-\left(q-r_{n} A q\right)\right\|^{2}+\frac{1}{2}\left\|y_{n}-q\right\|^{2} \\
& -\frac{1}{2}\left\|\left(x_{n}-r_{n} A x_{n}\right)-\left(q-r_{n} A q\right)-\left(y_{n}-q\right)\right\|^{2} \\
\leq & \frac{1}{2}\left[\left\|x_{n}-q\right\|^{2}+\left\|y_{n}-q\right\|^{2}-\left\|\left(x_{n}-y_{n}\right)-2 r_{n}\left(A x_{n}-A q\right)\right\|^{2}\right] \\
= & \frac{1}{2}\left[\left\|x_{n}-q\right\|^{2}+\left\|y_{n}-q\right\|^{2}-\left\|x_{n}-y_{n}\right\|^{2}+2 r_{n}\left\langle x_{n}-y_{n}, A x_{n}-A q\right\rangle\right. \\
& \left.-r_{n}^{2}\left\|A x_{n}-A q\right\|^{2}\right] .
\end{aligned}
$$

It follows that

$$
\left\|y_{n}-q\right\|^{2} \leq\left\|x_{n}-q\right\|^{2}-\left\|x_{n}-y_{n}\right\|^{2}+2 r_{n}\left\langle x_{n}-y_{n}, A x_{n}-A q\right\rangle .
$$

From (8), (17), and (18) we have

$$
\begin{aligned}
\left\|t_{n}-q\right\|^{2}= & \left\|a_{n}\left(S z_{n}-q\right)+\left(1-a_{n}\right)\left(T y_{n}-q\right)\right\|^{2} \\
\leq & a_{n}\left\|S z_{n}-q\right\|^{2}+\left(1-a_{n}\right)\left\|T y_{n}-q\right\|^{2} \\
\leq & a_{n}\left\|z_{n}-q\right\|^{2}+\left(1-a_{n}\right)\left\|y_{n}-q\right\|^{2} \\
\leq & a_{n}\left(\mu_{n}\left\|x_{n}-q\right\|^{2}+\left(1-\mu_{n}\right)\left\|w_{n}-q\right\|^{2}\right)+\left(1-a_{n}\right)\left\|y_{n}-q\right\|^{2} \\
\leq & a_{n}\left(\mu_{n}\left\|x_{n}-q\right\|^{2}+\left(1-\mu_{n}\right)\left(\left\|y_{n}-q\right\|^{2}-\left\|y_{n}-w_{n}\right\|^{2}\right)\right) \\
& +\left(1-\mu_{n}\right) a_{n} 2 \lambda_{n}\left\|y_{n}-w_{n}\right\|\left\|M y_{n}-M q\right\| \\
& +\left(1-a_{n}\right)\left(\left\|x_{n}-q\right\|^{2}-\left\|x_{n}-y_{n}\right\|^{2}+2 r_{n}\left\langle x_{n}-y_{n}, A x_{n}-A q\right\rangle\right) \\
\leq & \left\|x_{n}-q\right\|^{2}-a_{n}\left(1-\mu_{n}\right)\left\|y_{n}-w_{n}\right\|^{2} \\
& +\left(1-\mu_{n}\right) a_{n} 2 \lambda_{n}\left\|y_{n}-w_{n}\right\|\left\|M y_{n}-M q\right\| \\
& +\left(1-a_{n}\right)\left(2 r_{n}\left\|x_{n}-y_{n}\right\|\left\|A x_{n}-A q\right\|-\left\|x_{n}-y_{n}\right\|^{2}\right) .
\end{aligned}
$$

By using (6) and last inequality we see that

$$
\begin{aligned}
\left\|x_{n+1}-q\right\|^{2}= & \left\|P_{C}\left[b_{n} f\left(x_{n}\right)+\left(1-b_{n}\right) t_{n}\right]-P_{C}(q)\right\|^{2} \\
\leq & b_{n}\left\|f\left(x_{n}\right)-q\right\|^{2}+\left(1-b_{n}\right)\left\|t_{n}-q\right\|^{2} \\
\leq & b_{n}\left\|f\left(x_{n}\right)-q\right\|^{2}+\left(1-b_{n}\right)\left(\left\|x_{n}-q\right\|^{2}-a_{n}\left(1-\mu_{n}\right)\left\|y_{n}-w_{n}\right\|^{2}\right. \\
& +\left(1-\mu_{n}\right) a_{n} 2 \lambda_{n}\left\|y_{n}-w_{n}\right\|\left\|M y_{n}-M q\right\| \\
& \left.+\left(1-a_{n}\right)\left(2 r_{n}\left\|x_{n}-y_{n}\right\|\left\|A x_{n}-A q\right\|-\left\|x_{n}-y_{n}\right\|^{2}\right)\right) .
\end{aligned}
$$

Thus

$$
\begin{aligned}
\left(1-b_{n}\right)\left(1-a_{n}\right)\left\|x_{n}-y_{n}\right\|^{2} \leq & b_{n}\left\|f\left(x_{n}\right)-q\right\|^{2}+\left\|x_{n}-q\right\|^{2}-\left\|x_{n+1}-q\right\|^{2} \\
& +\left(1-\mu_{n}\right) a_{n} 2 \lambda_{n}\left\|y_{n}-w_{n}\right\|\left\|M y_{n}-M q\right\|-\left\|y_{n}-w_{n}\right\|^{2} \\
& +\left(1-a_{n}\right)\left(2 r_{n}\left\|x_{n}-y_{n}\right\|\left\|A x_{n}-A q\right\|-\left\|x_{n}-y_{n}\right\|^{2}\right)
\end{aligned}
$$


and

$$
\begin{aligned}
\left(1-b_{n}\right)\left(1-\mu_{n}\right) a_{n}\left\|y_{n}-w_{n}\right\|^{2} \leq & b_{n}\left\|f\left(x_{n}\right)-q\right\|^{2}+\left\|x_{n}-q\right\|^{2}-\left\|x_{n+1}-q\right\|^{2} \\
& +\left(1-\mu_{n}\right) a_{n} 2 \lambda_{n}\left\|y_{n}-w_{n}\right\|\left\|M y_{n}-M q\right\| \\
& +\left(1-a_{n}\right) 2 r_{n}\left\|x_{n}-y_{n}\right\|\left\|A x_{n}-A q\right\| .
\end{aligned}
$$

From $\left(d_{3}\right)$, (15), and $\lim _{n \rightarrow \infty}\left\|x_{n}-x_{n+1}\right\|=0$ we find $\lim _{n \rightarrow \infty}\left\|x_{n}-y_{n}\right\|=0$ and also $\lim _{n \rightarrow \infty}\left\|y_{n}-w_{n}\right\|=0$. Since $\left\|x_{n}-w_{n}\right\| \leq\left\|x_{n}-y_{n}\right\|+\left\|y_{n}-w_{n}\right\|$, we get $\left\|x_{n}-w_{n}\right\| \rightarrow 0$. Consequently, by using (6) we get $\lim _{n \rightarrow \infty}\left\|z_{n}-x_{n}\right\|=\lim _{n \rightarrow \infty}\left(1-\mu_{n}\right)\left\|w_{n}-x_{n}\right\|=0$. Moreover, from (14) and (16) we get $\left\|z_{n}-S z_{n}\right\| \leq\left\|z_{n}-x_{n}\right\|+\left\|x_{n}-t_{n}\right\|+\left\|t_{n}-S z_{n}\right\| \rightarrow 0$ and $\left\|y_{n}-T y_{n}\right\| \leq\left\|y_{n}-x_{n}\right\|+\left\|x_{n}-t_{n}\right\|+\left\|t_{n}-T y_{n}\right\| \rightarrow 0$. Hence

$$
\begin{aligned}
\left\|x_{n}-S x_{n}\right\| & \leq\left\|x_{n}-z_{n}\right\|+\left\|z_{n}-S z_{n}\right\|+\left\|S z_{n}-S x_{n}\right\| \\
& \leq\left\|x_{n}-z_{n}\right\|+\left\|z_{n}-S z_{n}\right\|+\left\|z_{n}-x_{n}\right\| \rightarrow 0
\end{aligned}
$$

and

$$
\begin{aligned}
\left\|x_{n}-T x_{n}\right\| & \leq\left\|x_{n}-y_{n}\right\|+\left\|y_{n}-T y_{n}\right\|+\left\|T y_{n}-T x_{n}\right\| \\
& \leq\left\|x_{n}-y_{n}\right\|+\left\|y_{n}-T y_{n}\right\|+\left\|y_{n}-x_{n}\right\| \rightarrow 0
\end{aligned}
$$

which implies

$$
\lim _{n \rightarrow \infty}\left\|x_{n}-T x_{n}\right\|=\lim _{n \rightarrow \infty}\left\|x_{n}-S x_{n}\right\|=0
$$

Now we show that $\limsup _{n \rightarrow \infty}\left\langle f(q)-q, x_{n}-q\right\rangle \leq 0$, where $q=P_{\Omega} f(q)$. The existence of $q$ is justified since $P_{\Omega}$ is nonexpansive and $f$ is a contraction. Hence $P_{\Omega} \circ f$ is a contraction and so has a fixed point. We can choose a subsequence $\left\{x_{n_{i}}\right\}$ of $\left\{x_{n}\right\}$ such that

$$
\limsup _{n \rightarrow \infty}\left\langle f(q)-q, x_{n}-q\right\rangle=\lim _{i \rightarrow \infty}\left\langle f(q)-q, x_{n_{i}}-q\right\rangle .
$$

Since $\left\{x_{n_{i}}\right\}$ is bounded, there exists a subsequence $\left\{x_{n_{i_{j}}}\right\}$ of $\left\{x_{n_{i}}\right\}$ that converges weakly to $v$. Without loss of generality, we can assume that $x_{n_{i}} \rightarrow v$. Since $\left\|x_{n}-y_{n}\right\| \rightarrow 0$ and $\left\|x_{n}-z_{n}\right\| \rightarrow 0$, we find $y_{n_{i}} \rightarrow v$ and $y_{n_{i}} \rightarrow v$. Since $\left\{y_{n_{i}}\right\}$ and $\left\{z_{n_{i}}\right\}$ lie in $C$ and $C$ is closed and convex, we obtain $v \in C$. It is easy to check that $v \in F(T)$ and $v \in F(S)$. By using (19) and Lemma 2.3 we get $v \in F(T) \cap F(S)$. Now we show $v \in \operatorname{GEP}(F)$. Since $y_{n}=T_{r_{n}}\left(x_{n}-r_{n} A x_{n}\right)$, we obtain

$$
F\left(y_{n}, y\right)+\left\langle A x_{n}, y-y_{n}\right\rangle+\frac{1}{r_{n}}\left\langle y-y_{n}, y_{n}-x_{n}\right\rangle \geq 0 \quad(\forall y \in C) .
$$

From $\left(A_{2}\right)$ we get $\left\langle A x_{n}, y-y_{n}\right\rangle+\frac{1}{r_{n}}\left\langle y-y_{n}, y_{n}-x_{n}\right\rangle \geq F\left(y, y_{n}\right)$ for all $y \in C$. Hence

$$
\left\langle A x_{n_{i}}, y-y_{n_{i}}\right\rangle+\left\langle y-y_{n_{i}}, \frac{y_{n_{i}}-x_{n_{i}}}{r_{n_{i}}}\right\rangle \geq F\left(y, y_{n_{i}}\right)
$$


for all $y \in C$. For $0<t \leq 1$ and $y \in C$, put $y_{t}=t y+(1-t) v$. Since $y \in C$ and $v \in C$, we obtain $y_{t} \in C$. From (21) we conclude that

$$
\begin{aligned}
\left\langle y_{t}-y_{n_{i}}, A y_{t}\right\rangle \geq & \left\langle y_{t}-y_{n_{i}}, A y_{t}\right\rangle-\left\langle y_{t}-y_{n_{i}}, A x_{n_{i}}\right\rangle \\
& -\left\langle y_{t}-y_{n_{i}}, \frac{y_{n_{i}}-x_{n_{i}}}{r_{n_{i}}}\right\rangle+F\left(y_{t}, y_{n_{i}}\right) \\
= & \left\langle y_{t}-y_{n_{i}}, A y_{t}-A y_{n_{i}}\right\rangle+\left\langle y_{t}-y_{n_{i}}, A y_{n_{i}}-A x_{n_{i}}\right\rangle \\
& -\left\langle y_{t}-y_{n_{i}}, \frac{y_{n_{i}}-x_{n_{i}}}{r_{n_{i}}}\right\rangle+F\left(y_{t}, y_{n_{i}}\right) .
\end{aligned}
$$

Since $\left\|y_{n_{i}}-x_{n_{i}}\right\| \rightarrow 0$, we have $\left\|A y_{n_{i}}-A x_{n_{i}}\right\| \rightarrow 0$. Further, from the inverse-strongly monotonicity of $A$ we have $\left\langle y_{t}-y_{n_{i}}, A y_{t}-A y_{n_{i}}\right\rangle \geq 0$. By using $\left(A_{4}\right), \frac{y_{n_{i}}-x_{n_{i}}}{r_{n_{i}}} \rightarrow 0$, and $y_{n_{i}} \rightarrow v$ we get $\left\langle y_{t}-v, A y_{t}\right\rangle \geq F\left(y_{t}, v\right)$. From $\left(A_{1}\right)-\left(A_{4}\right)$ we have

$$
\begin{aligned}
0 & =F\left(y_{t}, y_{t}\right)=t F\left(y_{t}, y\right)+(1-t) F\left(y_{t}, v\right) \\
& \leq t F\left(y_{t}, y\right)+(1-t)\left\langle y_{t}-v, A y_{t}\right\rangle \\
& =t F\left(y_{t}, y\right)+(1-t) t\left\langle y-v, A y_{t}\right\rangle,
\end{aligned}
$$

and so $0 \leq F\left(y_{t}, y\right)+(1-t)\left\langle y-v, A y_{t}\right\rangle$. Thus $F(v, y)+(1-t)\langle y-v, A v\rangle \geq 0$ for all $y \in C$. This implies that $v \in \operatorname{GEP}(F, A)$. Finally, we show $v \in(M+B)^{-1}(0)$. Choose a subsequence $\left\{\lambda_{n_{i_{j}}}\right\}$ of $\left\{\lambda_{n_{i}}\right\}$ such that $\lambda_{n_{i_{j}}} \rightarrow \tilde{\lambda} \in[c, d]$. Without loss of generality, assume that $\lambda_{n_{i}} \rightarrow \tilde{\lambda}$. By using Lemma 2.6 we obtain

$$
\begin{aligned}
\left\|y_{n_{i}}-J_{\tilde{\lambda}}^{B}(I-\tilde{\lambda} M) y_{n_{i}}\right\| \leq & \left\|y_{n_{i}}-z_{n_{i}}\right\|+\left\|z_{n_{i}}-\left(\mu_{n_{i}} x_{n_{i}}+\left(1-\mu_{n_{i}}\right) J_{\tilde{\lambda}}^{B}(I-\tilde{\lambda} M) y_{n_{i}}\right)\right\| \\
& +\left\|\left(\mu_{n_{i}} x_{n_{i}}+\left(1-\mu_{n_{i}}\right) J_{\tilde{\lambda}}^{B}(I-\tilde{\lambda} M) y_{n_{i}}\right)-J_{\tilde{\lambda}}^{B}(I-\tilde{\lambda} M) y_{n_{i}}\right\| \\
\leq & \left.\left\|y_{n_{i}}-z_{n_{i}}\right\|+\left(1-\mu_{n_{i}}\right) \| J_{\lambda_{n_{i}}}^{B}\left(I-\lambda n_{n_{i}} M\right) y_{n_{i}}-J_{\tilde{\lambda}}^{B}(I-\tilde{\lambda} M) y_{n_{i}}\right) \| \\
& +\mu_{n_{i}}\left\|x_{n_{i}}-J_{\tilde{\lambda}}^{B}(I-\tilde{\lambda} M) y_{n_{i}}\right\| \\
\leq & \left\|y_{n_{i}}-z_{n_{i}}\right\|+\left(1-\mu_{n_{i}}\right)\left[\left\|J_{\lambda_{n_{i}}}^{B}\left(I-\lambda_{n_{i}} M\right) y_{n_{i}}-J_{\lambda_{n_{i}}}^{B}(I-\tilde{\lambda} M) y_{n_{i}}\right\|\right. \\
& \left.+\left\|J_{\lambda_{n_{i}}}^{B}(I-\tilde{\lambda} M) y_{n_{i}}-J_{\tilde{\lambda}}^{B}(I-\tilde{\lambda} M) y_{n_{i}}\right\|\right] \\
& +\mu_{n_{i}}\left\|x_{n_{i}}-J_{\tilde{\lambda}}^{B}(I-\tilde{\lambda} M) y_{n_{i}}\right\| \\
\leq & \left\|y_{n_{i}}-z_{n_{i}}\right\|+\left(1-\mu_{n_{i}}\right)\left|\lambda_{n_{i}}-\tilde{\lambda}\right|\left\|M y_{n_{i}}\right\| \\
& +\left(1-\mu_{n_{i}}\right)\left|\frac{\lambda n_{n_{i}}-\tilde{\lambda}}{\tilde{\lambda}}\right|\left\|J_{\tilde{\lambda}}^{B}(I-\tilde{\lambda} M) y_{n_{i}}-(I-\tilde{\lambda} M) y_{n_{i}}\right\| \\
& +\mu_{n_{i}}\left[\left\|x_{n_{i}}-y_{n_{i}}\right\|+\left\|y_{n_{i}}-J_{\tilde{\lambda}}^{B}(I-\tilde{\lambda} M) y_{n_{i}}\right\|\right] .
\end{aligned}
$$

Thus

$$
\begin{aligned}
\left(1-\mu_{n_{i}}\right)\left\|y_{n_{i}}-J_{\tilde{\lambda}}^{B}(I-\tilde{\lambda} M) y_{n_{i}}\right\| \leq & \left\|y_{n_{i}}-z_{n_{i}}\right\|+\left(1-\mu_{n_{i}}\right)\left|\lambda_{n_{i}}-\tilde{\lambda}\right|\left\|M y_{n_{i}}\right\| \\
& +\left(1-\mu_{n_{i}}\right)\left|\frac{\lambda_{n_{i}}-\tilde{\lambda}}{\tilde{\lambda}}\right|\left\|J_{\tilde{\lambda}}^{B}(I-\tilde{\lambda} M) y_{n_{i}}-(I-\tilde{\lambda} M) y_{n_{i}}\right\| \\
& +\mu_{n_{i}}\left\|x_{n_{i}}-y_{n_{i}}\right\| .
\end{aligned}
$$


This implies that $\lim _{k \rightarrow \infty}\left\|y_{n_{i}}-J_{\tilde{\lambda}}^{B}(I-\tilde{\lambda} M) y_{n_{i}}\right\|=0$. Since $J_{\tilde{\lambda}}^{B}(I-\tilde{\lambda} M)$ is nonexpansive, it is demiclosed, and so $v \in F\left(J_{\tilde{\lambda}}^{B}(I-\tilde{\lambda} M)\right)$, that is, $v \in(M+B)^{-1}(0)$. This implies $v \in \Omega$. By using (20) we get $\limsup _{n \rightarrow \infty}\left\langle f(q)-q, x_{n}-q\right\rangle=\lim _{i \rightarrow \infty}\left\langle f(q)-q, x_{n_{i}}-q\right\rangle=\langle f(q)-q, v-q\rangle \leq 0$. Now we show that $x_{n} \rightarrow q$. From(8) and (9) we have

$$
\begin{aligned}
\left\|t_{n}-q\right\| & =\left\|\left(a_{n} S z_{n}+\left(1-a_{n}\right) T y_{n}\right)-q\right\| \\
& \leq a_{n}\left\|S z_{n}-q\right\|+\left(1-a_{n}\right)\left\|T y_{n}-q\right\| \\
& \leq a_{n}\left\|z_{n}-q\right\|+\left(1-a_{n}\right)\left\|y_{n}-q\right\| \leq\left\|x_{n}-q\right\| .
\end{aligned}
$$

Set $v_{n}=b_{n} f\left(x_{n}\right)+\left(1-b_{n}\right) t_{n}$ for all $n \geq 1$. By using (6) and the property of metric projection we obtain

$$
\begin{aligned}
\left\|x_{n+1}-q\right\|^{2}= & \left\langle P_{C}\left(v_{n}\right)-v_{n}, P_{C}\left(v_{n}\right)-q\right\rangle+\left\langle v_{n}-q, x_{n+1}-q\right\rangle \\
\leq & \left\langle\left(b_{n} f\left(x_{n}\right)+\left(1-b_{n}\right) t_{n}\right)-q, x_{n+1}-q\right\rangle \\
= & b_{n}\left\langle f\left(x_{n}\right)-q, x_{n+1}-q\right\rangle+\left(1-b_{n}\right)\left\langle t_{n}-q, x_{n+1}-q\right\rangle \\
\leq & b_{n}\left\langle f\left(x_{n}\right)-f(q), x_{n+1}-q\right\rangle+b_{n}\left\langle f(q)-q, x_{n+1}-q\right\rangle \\
& +\left(1-b_{n}\right)\left\|t_{n}-q\right\|\left\|x_{n+1}-q\right\| \\
\leq & b_{n} \rho\left\|x_{n}-q\right\|\left\|x_{n+1}-q\right\|+b_{n}\left\langle f(q)-q, x_{n+1}-q\right\rangle \\
& +\left(1-b_{n}\right)\left\|x_{n}-q\right\|\left\|x_{n+1}-q\right\| \\
\leq & \left(1-b_{n}(1-\rho)\right)\left\|x_{n}-q\right\|\left\|x_{n+1}-q\right\|+b_{n}\left\langle f(q)-q, x_{n+1}-q\right\rangle \\
\leq & \frac{\left(1-b_{n}(1-\rho)\right)}{2}\left(\left\|x_{n}-q\right\|^{2}+\left\|x_{n+1}-q\right\|^{2}\right)+b_{n}\left\langle f(q)-q, x_{n+1}-q\right\rangle,
\end{aligned}
$$

which implies that $\left\|x_{n+1}-q\right\|^{2} \leq\left(1-b_{n}(1-\rho)\right)\left\|x_{n}-q\right\|^{2}+2 b_{n}\left\langle f(q)-q, x_{n+1}-q\right\rangle$. Now by using $\left(d_{3}\right)$ and Lemma 2.4 we get $\lim _{n \rightarrow \infty}\left\|x_{n}-q\right\|=0$. This completes the proof.

Let $u \in C$ and $f(x)=u \in C$ for all $x$. By using Theorem 3.1 we obtain the following result.

Corollary 3.2 Let $C$ be a nonempty closed convex subset of $H$, let $F$ be a bifunction from $C \times C$ to $\mathbb{R}$ satisfying $\left(A_{1}\right)-\left(A_{4}\right)$, let $A$ be an $\alpha$-inverse strongly monotone mapping from $C$ into $H$, let $M$ be a $\beta$-inverse strongly monotone map from $C$ into $H$, and let $B$ be a maximal monotone operator on $H$ with domain contained in $C$. Assume that $S, T: C \rightarrow C$ are two nonexpansive mappings such that $\Omega=F(T) \cap F(S) \cap(M+B)^{-1}(0) \cap \operatorname{GEP}(F, A) \neq \emptyset$. Suppose that $\left\{b_{n}\right\},\left\{a_{n}\right\}$, and $\left\{\mu_{n}\right\}$ are some sequences in $(0,1)$ and that $\left\{x_{n}\right\},\left\{y_{n}\right\}$, and $\left\{z_{n}\right\}$ are the sequences generated by

$$
\left\{\begin{array}{l}
x_{1} \in C, \\
F\left(y_{n}, y\right)+\left\langle A x_{n}, y-y_{n}\right\rangle+\frac{1}{r_{n}}\left\langle y-y_{n}, y_{n}-x_{n}\right\rangle \geq 0 \quad(\forall y \in C), \\
\left.z_{n}=\mu_{n} x_{n}+\left(1-\mu_{n}\right)\right)_{\lambda_{n}}^{B}\left(y_{n}-\lambda_{n} M y_{n}\right), \\
x_{n+1}=P_{C}\left[b_{n} u+\left(1-b_{n}\right)\left(a_{n} S z_{n}+\left(1-a_{n}\right) T y_{n}\right)\right] \quad(\forall n \geq 1) .
\end{array}\right.
$$

If $\left(d_{1}\right)-\left(d_{4}\right)$ hold, then the sequence $\left\{x_{n}\right\}$ converges strongly to a point $q \in \Omega$, which is the unique solution to the variational inequality $\langle q-u, x-q\rangle \geq 0$ for all $x \in \Omega$. 
Corollary 3.3 Let $C$ be a nonempty closed convex subset of $H$, let $F$ be a bifunction from $C \times C$ to $\mathbb{R}$ satisfying $\left(A_{1}\right)-\left(A_{4}\right)$, let $A$ be an $\alpha$-inverse strongly monotone mapping from $C$ into $H$, let $M$ be a $\beta$-inverse strongly monotone map from $C$ into $H$, and let $B$ be a maximal monotone operator on $H$ with domain contained in $C$. Assume that $S, T: C \rightarrow C$ are two nonexpansive mappings such that $\Omega=F(T) \cap F(S) \cap(M+B)^{-1}(0) \cap \operatorname{GEP}(F, A) \neq \emptyset$. Suppose that $\left\{b_{n}\right\},\left\{a_{n}\right\}$, and $\left\{\mu_{n}\right\}$ are some sequences in $(0,1)$ and that $\left\{x_{n}\right\},\left\{y_{n}\right\}$, and $\left\{z_{n}\right\}$ are the sequences generated by

$$
\left\{\begin{array}{l}
x_{1} \in C, \\
F\left(y_{n}, y\right)+\left\langle A x_{n}, y-y_{n}\right\rangle+\frac{1}{r_{n}}\left\langle y-y_{n}, y_{n}-x_{n}\right\rangle \geq 0 \quad(\forall y \in C), \\
z_{n}=\mu_{n} x_{n}+\left(1-\mu_{n}\right) J_{\lambda_{n}}^{B}\left(y_{n}-\lambda_{n} M y_{n}\right), \\
x_{n+1}=P_{C}\left[\left(1-b_{n}\right)\left(a_{n} S z_{n}+\left(1-a_{n}\right) T y_{n}\right)\right] \quad(\forall n \geq 1) .
\end{array}\right.
$$

If $\left(d_{1}\right)-\left(d_{4}\right)$ hold, then the sequence $\left\{x_{n}\right\}$ converges strongly to a point $q=P_{\Omega}(0)$, which is the minimum norm element in $\Omega$.

Proof In Theorem 3.1, put $f(x)=0$ for all $x$. Note that $x_{n} \rightarrow q=P_{\Omega}(0)$ and $P_{\Omega}(0)$ is the minimum norm element in $\Omega$. Since $\langle(I-f) q, x-q\rangle \geq 0$, we get $\langle q, q-x\rangle \leq 0$ for all $x \in \Omega$, that is, $\|q\|^{2} \leq\langle q, x\rangle \leq\|x\|\|q\|$ for all $x \in \Omega$. Thus, the point $q$ is the unique solution to the quadratic minimization problem $q=\arg \min _{x \in \Omega}\|x\|^{2}$.

Let $I_{C}$ be the indicator function of $C$ defined by $I_{C}(x)=0$ for $x \in C$ and $I_{C}(x)=\infty$ otherwise. Recall that the subdifferential $\partial I_{C}$ is a maximal monotone operator. Note that $I_{C}$ is a proper lower semicontinuous convex function on $H$. The resolvent $J_{r}^{\partial I_{C}}$ of $\partial I_{C}$ for $r$ is $P_{C}$, and $\mathrm{VI}(C, M)=\left(M+\partial I_{C}\right)^{-1}(0)$, where $M$ is an inverse strongly monotone mapping from $C$ into $H$ [35].

Theorem 3.4 Let $C$ be a nonempty closed convex subset of $H$, let $F$ be a bifunction from $C \times C$ to $\mathbb{R}$ satisfying $\left(A_{1}\right)-\left(A_{4}\right)$, let $A$ be an $\alpha$-inverse strongly monotone mapping from $C$ into $H$, let $M$ be a $\beta$-inverse strongly monotone map from $C$ into $H$, and let $B$ be a maximal monotone operator on $H$ with domain contained in $C$. Assume that $S, T: C \rightarrow C$ are two nonexpansive mappings such that $\Omega=F(T) \cap F(S) \cap \operatorname{VI}(C, M) \cap \operatorname{GEP}(F, A) \neq \emptyset$ and $f: C \rightarrow$ $C$ is a contraction map with the constant $\rho \in(0,1)$. Suppose that $\left\{b_{n}\right\},\left\{a_{n}\right\}$, and $\left\{\mu_{n}\right\}$ are some sequences in $(0,1)$ and that $\left\{x_{n}\right\},\left\{y_{n}\right\}$, and $\left\{z_{n}\right\}$ are the sequences generated by

$$
\left\{\begin{array}{l}
x_{1} \in C, \\
F\left(y_{n}, y\right)+\left\langle A x_{n}, y-y_{n}\right\rangle+\frac{1}{r_{n}}\left\langle y-y_{n}, y_{n}-x_{n}\right\rangle \geq 0 \quad(\forall y \in C), \\
z_{n}=\mu_{n} x_{n}+\left(1-\mu_{n}\right) P_{C}\left(y_{n}-\lambda_{n} M y_{n}\right), \\
x_{n+1}=P_{C}\left[b_{n} f\left(x_{n}\right)+\left(1-b_{n}\right)\left(a_{n} S z_{n}+\left(1-a_{n}\right) T y_{n}\right)\right] \quad(\forall n \geq 1) .
\end{array}\right.
$$

If $\left(d_{1}\right)-\left(d_{4}\right)$ hold, then $\left\{x_{n}\right\}$ converges strongly to a point $p \in \Omega$, which is the unique solution to the variational inequality $\langle(I-f) p, x-p\rangle \geq 0$ for all $x \in \Omega$.

Proof If $B=\partial I_{C}$ in Theorem 3.1, then $J_{\lambda_{n}}=P_{C}$ for all $\lambda_{n}>0$. This completes the proof.

Note that Theorem 3.4 reduces the results of $[1,36]$. 
Theorem 3.5 Let $C$ be a nonempty closed convex subset of $H$, let $F$ be a bifunction from $C \times C$ to $\mathbb{R}$ satisfying $\left(A_{1}\right)-\left(A_{4}\right)$, let $A$ be an $\alpha$-inverse strongly monotone mapping from $C$ into $H$, let $M$ be a $\beta$-inverse strongly monotone map from $C$ into $H$, and let $B$ be a maximal monotone operator on $H$ with domain contained in $C$. Assume that $S, T: C \rightarrow C$ are two nonexpansive mappings such that $\Omega=F(T) \cap F(S) \cap \operatorname{VI}(C, M) \neq \emptyset$. Suppose that $\left\{b_{n}\right\},\left\{a_{n}\right\}$, and $\left\{\mu_{n}\right\}$ are some sequences in $(0,1)$ and that $\left\{x_{n}\right\}$ and $\left\{z_{n}\right\}$ are some sequences generated by

$$
\left\{\begin{array}{l}
x_{1} \in C, \\
z_{n}=\mu_{n} x_{n}+\left(1-\mu_{n}\right) P_{C}\left(x_{n}-\lambda_{n} M x_{n}\right), \\
x_{n+1}=P_{C}\left[b_{n} f\left(x_{n}\right)+\left(1-b_{n}\right)\left(a_{n} S z_{n}+\left(1-a_{n}\right) T x_{n}\right)\right] \quad(\forall n \geq 1) .
\end{array}\right.
$$

If $\left(d_{1}\right)-\left(d_{4}\right)$ hold, then $\left\{x_{n}\right\}$ converges strongly to a point $p \in \Omega$, which is the unique solution to the variational inequality $\langle(I-f) p, x-p\rangle \geq 0$ for all $x \in \Omega$.

Proof Put $F=A=0, B=\partial I_{C}$, and $r_{n}=1$ for all $n$ in Theorem 3.1. Since $J_{\lambda_{n}}=P_{C}$ for all $\lambda_{n}>0$, we obtain the desired result.

We can see that Theorem 3.5 extends Theorem 11 in [37]. The next result reduces the related result of [38].

Theorem 3.6 Let $C$ be a nonempty closed convex subset of $H$, let $F$ be a bifunction from $C \times C$ to $\mathbb{R}$ satisfying $\left(A_{1}\right)-\left(A_{4}\right)$, let $M$ be a $\beta$-inverse strongly monotone map from $C$ into $H$, and let $B$ be a maximal monotone operator on $H$ with domain contained in $C$. Assume that $S: C \rightarrow C$ is a nonexpansive mapping such that $\Omega=F(S) \cap \operatorname{VI}(C, M) \neq \emptyset$ and $f: C \rightarrow C$ is a contraction map with the constant $\rho \in(0,1)$. Suppose that $\left\{b_{n}\right\}$ and $\left\{\mu_{n}\right\}$ are some sequences in $(0,1)$ and that $\left\{x_{n}\right\}$ and $\left\{z_{n}\right\}$ are the sequences generated by

$$
\left\{\begin{array}{l}
x_{1} \in C \\
z_{n}=\mu_{n} x_{n}+\left(1-\mu_{n}\right) P_{C}\left(x_{n}-\lambda_{n} M x_{n}\right) \\
x_{n+1}=P_{C}\left[b_{n} f\left(x_{n}\right)+\left(1-b_{n}\right) S z_{n}\right] \quad(\forall n \geq 1) .
\end{array}\right.
$$

If $\left(d_{1}\right)-\left(d_{4}\right)$ hold, then $\left\{x_{n}\right\}$ converges strongly to a point $p \in \Omega$, which is the unique solution to the variational inequality $\langle(I-f) p, x-p\rangle \geq 0$ for all $x \in \Omega$.

Proof Put $F=A=0, B=\partial I_{C}, T=I$, and $r_{n}=1$ for all $n$ in Theorem 3.1. Since $J_{\lambda_{n}}=P_{C}$ for all $\lambda_{n}>0$, we obtain the desired result.

Theorem 3.7 Let $C$ be a nonempty closed convex subset of $H$, let $F$ be a bifunction from $C \times C$ to $\mathbb{R}$ satisfying $\left(A_{1}\right)-\left(A_{4}\right)$, let $A$ be an $\alpha$-inverse strongly monotone mapping from $C$ into $H$, and let $\psi: C \rightarrow C$ be a $\beta$-strict pseudo-contraction. Assume that $S, T: C \rightarrow C$ are two nonexpansive mappings such that $\Omega=F(T) \cap F(S) \cap F(\psi) \neq \emptyset$, and $f: C \rightarrow C$ is a contraction map with the constant $\rho \in(0,1)$. Suppose that $\left\{b_{n}\right\},\left\{a_{n}\right\}$ and $\left\{\mu_{n}\right\}$ are some 
sequences in $(0,1)$ and that $\left\{x_{n}\right\}$ and $\left\{z_{n}\right\}$ are the sequences generated by

$$
\left\{\begin{array}{l}
x_{1} \in C \\
z_{n}=\mu_{n} x_{n}+\left(1-\mu_{n}\right)\left(\left(1-\lambda_{n}\right) x_{n}+\lambda_{n} \psi x_{n}\right), \\
x_{n+1}=P_{C}\left[b_{n} f\left(x_{n}\right)+\left(1-b_{n}\right)\left(a_{n} S z_{n}+\left(1-a_{n}\right) T x_{n}\right)\right] \quad(\forall n \geq 1) .
\end{array}\right.
$$

If $\left(d_{1}\right)-\left(d_{4}\right)$ hold and $0<c<\lambda_{n}<d<1-\beta$ for all $n$, then $\left\{x_{n}\right\}$ converges strongly to a point $p \in \Omega$, which is the unique solution to the variational inequality $\langle(I-f) p, x-p\rangle \geq 0$ for all $x \in \Omega$.

Proof Put $F=A=0, r_{n}=1$, and $M=I-\psi$. Then $M$ is $\frac{1-\beta}{2}$-inverse-strongly monotone map, $F(\psi)=V I(C, M)$, and $P_{C}\left(x_{n}-\lambda_{n} M x_{n}\right)=\left(1-\lambda_{n}\right) x_{n}+\lambda_{n} \psi x_{n}$ for all $n$. Now by using Theorem 3.5 we obtain the desired result.

Note that Theorem 3.7 reduces the result of [39].

Theorem 3.8 Let $C$ be a nonempty closed convex subset of $H$, let $F$ be a bifunction from $C \times C$ to $\mathbb{R}$ satisfying $\left(A_{1}\right)-\left(A_{4}\right)$, let $A$ be an $\alpha$-inverse strongly monotone mapping from $C$ into $H$, and let $\psi: C \rightarrow C$ be a $\beta$-strict pseudo-contraction. Assume that $S, T: C \rightarrow C$ are two nonexpansive mappings such that $\Omega=F(T) \cap F(S) \cap F(\psi) \cap \operatorname{GEP}(F, A) \neq \emptyset$ and $f: C \rightarrow C$ is a contraction map with the constant $\rho \in(0,1)$. Suppose that $\left\{b_{n}\right\},\left\{a_{n}\right\}$, and $\left\{\mu_{n}\right\}$ are some sequences in $(0,1)$ and that $\left\{x_{n}\right\},\left\{y_{n}\right\}$, and $\left\{z_{n}\right\}$ are the sequences generated by

$$
\left\{\begin{array}{l}
x_{1} \in C, \\
F\left(y_{n}, y\right)+\left\langle A x_{n}, y-y_{n}\right\rangle+\frac{1}{r_{n}}\left\langle y-y_{n}, y_{n}-x_{n}\right\rangle \geq 0 \quad(\forall y \in C), \\
z_{n}=\mu_{n} x_{n}+\left(1-\mu_{n}\right)\left(\left(1-\lambda_{n}\right) y_{n}+\lambda_{n} \psi y_{n}\right), \\
x_{n+1}=P_{C}\left[b_{n} f\left(x_{n}\right)+\left(1-b_{n}\right)\left(a_{n} S z_{n}+\left(1-a_{n}\right) T y_{n}\right)\right] \quad(\forall n \geq 1) .
\end{array}\right.
$$

If $\left(d_{1}\right)-\left(d_{4}\right)$ hold and $0<c<\lambda_{n}<d<1-\beta$ for all $n$, then $\left\{x_{n}\right\}$ converges strongly to a point $p \in \Omega$, which is the unique solution to the variational inequality $\langle(I-f) p, x-p\rangle \geq 0$ for all $x \in \Omega$.

Proof Put $M=I-\psi$. Then $M$ is $\frac{1-\beta}{2}$-inverse-strongly monotone mapping, $F(\psi)=$ $V I(C, M)$, and $P_{C}\left(y_{n}-\lambda_{n} M y_{n}\right)=\left(1-\lambda_{n}\right) y_{n}+\lambda_{n} \psi y_{n}$ for all $n$. By using Theorem 3.1 we obtain the desired result.

We can check that Theorem 3.8 reduces the result of [40].

Theorem 3.9 Let $C$ be a nonempty closed convex subset of $H$, let $M$ be a $\beta$-inverse strongly monotone map from $C$ into $H$, and let $B$ be a maximal monotone operator on $H$ with domain contained in $C$. Assume that $S, T: C \rightarrow C$ are two nonexpansive mappings such that $\Omega=F(T) \cap F(S) \cap(M+B)^{-1}(0) \neq \emptyset$. Suppose that $\left\{b_{n}\right\},\left\{a_{n}\right\}$, and $\left\{\mu_{n}\right\}$ are some sequences 
in $(0,1)$ and that $\left\{x_{n}\right\}$ and $\left\{z_{n}\right\}$ are the sequences generated by

$$
\left\{\begin{array}{l}
x_{1} \in C \\
z_{n}=\mu_{n} x_{n}+\left(1-\mu_{n}\right) J_{\lambda_{n}}^{B}\left(x_{n}-\lambda_{n} M x_{n}\right), \\
x_{n+1}=P_{C}\left[b_{n} f\left(x_{n}\right)+\left(1-b_{n}\right)\left(a_{n} S z_{n}+\left(1-a_{n}\right) T x_{n}\right)\right] \quad(\forall n \geq 1) .
\end{array}\right.
$$

If $\left(d_{1}\right)-\left(d_{4}\right)$ hold, then $\left\{x_{n}\right\}$ converges strongly to a point $p \in \Omega$, which is the unique solution to the variational inequality $\langle(I-f) p, x-p\rangle \geq 0$ for all $x \in \Omega$.

Proof It is sufficient put $F=A=0$ and $r_{n}=1$ for all $n$ in Theorem 3.1.

We can see that Theorem 3.9 reduces the result of [31]. Let $g: H \rightarrow \mathbb{R} \cup\{+\infty\}$ be a convex lower semicontinuous proper function. Put $B=\partial g$, where $\partial$ denotes subdifferential of $g$. Then $B$ is a maximal monotone operator, and $0 \in \partial f(x)$ is equivalent to $g\left(x^{\prime}\right)=\min _{x \in C} g(x)[24,26]$. Recall that the subdifferential of $g$ at $x$ is defined by

$$
\partial g(x):=\{v \in H: g(y) \geq g(x)+\langle v, y-x\rangle \text { for all } y \in H\} .
$$

Theorem 3.10 Let $C$ be a nonempty closed convex subset of $H$, let $F$ be a bifunction from $C \times C$ to $\mathbb{R}$ satisfying $\left(A_{1}\right)-\left(A_{4}\right)$, A be an $\alpha$-inverse strongly monotone mapping from $C$ into $H$, and let $g: H \rightarrow(-\infty,+\infty]$ be a proper convex lower semicontinuous function. Assume that $S, T: C \rightarrow C$ are two nonexpansive mappings such that $\Omega=F(T) \cap F(S) \cap(\partial f)^{-1}(0) \cap$ $\operatorname{GEP}(F, A) \neq \emptyset$. Suppose that $\left\{b_{n}\right\},\left\{a_{n}\right\}$, and $\left\{\mu_{n}\right\}$ be sequences in $(0,1)$ and that $\left\{x_{n}\right\},\left\{y_{n}\right\}$, $\left\{u_{n}\right\}$, and $\left\{z_{n}\right\}$ are the sequences generated by

$$
\left\{\begin{array}{l}
x_{1} \in C, \\
F\left(y_{n}, y\right)+\left\langle A x_{n}, y-y_{n}\right\rangle+\frac{1}{r_{n}}\left\langle y-y_{n}, y_{n}-x_{n}\right\rangle \geq 0 \quad(\forall y \in C), \\
u_{n}=\arg \min _{w \in H}\left\{g(w)+\frac{\left\|w-y_{n}\right\|^{2}}{2 \lambda_{n}}\right\}, \\
z_{n}=\mu_{n} x_{n}+\left(1-\mu_{n}\right) u_{n}, \\
x_{n+1}=P_{C}\left[b_{n} f\left(x_{n}\right)+\left(1-b_{n}\right)\left(a_{n} S z_{n}+\left(1-a_{n}\right) T y_{n}\right)\right] \quad(\forall n \geq 1) .
\end{array}\right.
$$

If $\left(d_{1}\right)-\left(d_{4}\right)$ hold and $0<c<\lambda_{n}<d<\infty$ for all $n$, then $\left\{x_{n}\right\}$ converges strongly to a point $p \in \Omega$, which is the unique solution to the variational inequality $\langle(I-f) p, x-p\rangle \geq 0$ for all $x \in \Omega$.

Proof Put $M=0$. Then by Theorem 3.1 the desired result immediately follows.

Put $A=0$ in Theorem 3.1. Then we obtain next theorem, which reduces the result of [41].

Theorem 3.11 Let $C$ be a nonempty closed convex subset of $H$, let $F$ be a bifunction from $C \times C$ to $\mathbb{R}$ satisfying $\left(A_{1}\right)-\left(A_{4}\right)$, let $M$ be a $\beta$-inverse strongly monotone map from $C$ into $H$, and let $B$ be a maximal monotone operator on $H$ with domain contained in $C$. Assume that $S, T: C \rightarrow C$ are two nonexpansive mappings such that $\Omega=F(T) \cap F(S) \cap(M+$ 
$B)^{-1}(0) \cap \operatorname{GEP}(F) \neq \emptyset$ and $f: C \rightarrow C$ is a contraction map with the constant $\rho \in(0,1)$. Suppose that $\left\{b_{n}\right\},\left\{a_{n}\right\}$, and $\left\{\mu_{n}\right\}$ are some sequences in $(0,1)$ and that $\left\{x_{n}\right\},\left\{y_{n}\right\}$, and $\left\{z_{n}\right\}$ are the sequences generated by

$$
\left\{\begin{array}{l}
x_{1} \in C \\
F\left(y_{n}, y\right)+\frac{1}{r_{n}}\left\langle y-y_{n}, y_{n}-x_{n}\right\rangle \geq 0 \quad \forall y \in C, \\
z_{n}=\mu_{n} x_{n}+\left(1-\mu_{n}\right) J_{\lambda_{n}}^{B}\left(y_{n}-\lambda_{n} M y_{n}\right), \\
x_{n+1}=P_{C}\left[b_{n} f\left(x_{n}\right)+\left(1-b_{n}\right)\left(a_{n} S z_{n}+\left(1-a_{n}\right) T y_{n}\right)\right] \quad(\forall n \geq 1) .
\end{array}\right.
$$

If $\left(d_{1}\right)-\left(d_{4}\right)$ hold and $0<c<\lambda_{n}<d<\infty$ for all $n$, then $\left\{x_{n}\right\}$ converges strongly to a point $p \in \Omega$, which is the unique solution to the variational inequality $\langle(I-f) p, x-p\rangle \geq 0$ for all $x \in \Omega$.

Here we provide an example to illustrate Theorem 3.1.

Example 3.1 Let $H=\mathbb{R}$ with Euclidean norm and usual Euclidean inner product. Put $C:=$ $[-1, \infty), S x=\frac{x}{2}, T x=\frac{x}{3}, B x=\log (x+1), M x=4 x, \beta=\frac{1}{4}, F(x, y)=y-x, \alpha=\frac{1}{3}$, and $A x=3 x-1$ for all $x$. It is clear that $S$ and $T$ are nonexpansive, $M$ is a $\beta$-inverse strongly monotone mapping, $B$ is a maximal monotone operator, $F$ is a bifunction from $C \times C$ to $\mathbb{R}$ satisfying $\left(A_{1}\right)-\left(A_{4}\right), A$ is an $\alpha$-inverse strongly monotone mapping, and $0 \in \Omega=F(T) \cap F(S) \cap(M+$ $B)^{-1}(0) \cap \operatorname{GEP}(F, A)$. Now by using Theorem 3.1 the sequence $\left\{x_{n}\right\}$ converges strongly to a point $q \in \Omega$, which is the unique solution to the variational inequality $\langle(I-f) q, x-q\rangle \geq 0$ for all $x \in \Omega$.

\section{Acknowledgements}

Research of both authors were supported by Azarbaijan Shahid Madani University. The authors express their gratitude to unknown referees for their helpful suggestions, which improved final version of this paper.

Funding

Not applicable.

Availability of data and materials

Not applicable.

Ethics approval and consent to participate

Not applicable.

Competing interests

The authors declare that they have no competing interests.

Consent for publication

Not applicable.

Authors' contributions

All authors contributed equally and significantly in this manuscript, and they read and approved the final manuscript.

Author details

${ }^{1}$ Institute of Research and Development, Duy Tan University, Da Nang, 550000, Vietnam. ${ }^{2}$ Faculty of Natural Sciences, Duy Tan University, Da Nang, 550000, Vietnam. ${ }^{3}$ Department of Medical Research, China Medical University Hospital, China Medical University, Taichung, Taiwan. ${ }^{4}$ Department of Mathematics, Azarbaijan Shahid Madani University, Tabriz, Iran.

\section{Publisher's Note}

Springer Nature remains neutral with regard to jurisdictional claims in published maps and institutional affiliations. 


\section{References}

1. Takahashi, S., Takahashi, W.: Strong convergence theorem for a generalized equilibrium problem and a nonexpansive mapping in a Hilbert space. Nonlinear Anal. 69, 1025-1033 (2008)

2. Blum, E., Oettli, W.: From optimization and variational inequalities to equilibrium problems. Math. Stud. 63, 123-145 (1994)

3. Hartman, P., Stampacchia, G.: On some nonlinear elliptic differential-functional equations. Acta Math. 115, 271-310 (1966)

4. Ceng, L.C., Petrusel, A., Yao, J.C., Yao, Y.: Hybrid viscosity extragradient method for systems of variational inequalities, fixed points of nonexpansive mappings, zero points of accretive operators in Banach spaces. Fixed Point Theory 19(2), 487-502 (2018). https://doi.org/10.24193/fpt-ro.2018.2.39

5. Reich, S., Sabach, S.: Three strong convergence theorems regarding iterative methods for solving equilibrium problems in reflexive Banach spaces. Contemp. Math. 568, 225-240 (2012)

6. Rezapour, S., Zakeri, S.H.: Implicit iterative algorithms for $\alpha$-inverse strongly accretive operators in Banach spaces. J. Nonlinear Convex Anal. 20(8), 1547-1560 (2019)

7. Rezapour, S., Zakeri, S.H.: Strong convergence theorem for $\delta$-inverse strongly accretive operator in Banach spaces Appl. Set-Valued Anal. Optim. 1(1), 39-52 (2019)

8. Shehu, Y.: An iterative method for fixed point problems, variational inclusions and generalized equilibrium problems. Math. Comput. Model. 54, 1394-1404 (2011)

9. Takahashi, W., Wen, C.F., Yao, J.C.: The shrinking projection method for a finite family of demi-metric mappings with variational inequality problems in a Hilbert space. Fixed Point Theory 19(1), 407-419 (2018). https://doi.org/10.24193/fpt-ro.2018.1.32

10. Yu, L., Song, J.: Strong convergence theorems for solutions of fixed point and variational inequality problems. J. Inequal. Appl. 2014, 215 (2014)

11. Rezapour, S., Yao, Y., Zakeri, S.H.: Strong convergence theorems for generalized mixed equilibrium problems, variational inequality problems and fixed point problems with pseudo-contractive mappings. J. Nonlinear Convex Anal. 21(5), 1097-1108 (2020)

12. Ceng, L.C., Petrusel, A., Yao, J.C.: Strong convergence of modified implicit iterative algorithms with perturbed mappings for continuous pseudo-contractive mappings. Appl. Math. Comput. 209, 162-176 (2009)

13. Gobel, K., Reich, S.: Uniform Convexity, Hyperbolic Geometry and Nonexpansive Mappings. Taylor \& Francis, London (1984)

14. Ishikawa, S.: Fixed points by a new iteration method. Proc. Am. Math. Soc. 44(1), 147-150 (1974)

15. Kammura, S., Takahashi, W.: Approximating solutions of maximal monotone operators in Hilbert spaces. J. Approx. Theory 106, 226-240 (2000)

16. Reich, S.: The fixed point property for nonexpansive mappings I. Am. Math. Mon. 83, 266-268 (1976)

17. Reich, S.: The fixed point property for nonexpansive mappings II. Am. Math. Mon. 87, 292-294 (1980)

18. Wang, Y.H., Rezapour, S., Zakeri, S.H.: Strong convergence theorems for Bregman relatively nonexpansive mappings and continuous monotone mapping. J. Nonlinear Convex Anal. 20(3), 551-564 (2019)

19. Zhou, $\mathrm{H}$.: Convergence theorems of fixed points for $k$-strict pseudo-contractions in Hilbert spaces. Nonlinear Anal. $69,456-462(2008)$

20. Farid, M.: The subgradient extragradient method for solving mixed equilibrium problems and fixed point problems in Hilbert spaces. J. Appl. Numer. Optim. 1(3), 335-345 (2019)

21. Qin, X., Yao, J.C.: A viscosity iterative method for a split feasibility problem. J. Nonlinear Convex Anal. 20(8), 1497-1506 (2019)

22. Shahzad, N., Zegeye, H.: Convergence theorems of common solutions for fixed point, variational inequality and equilibrium problems. J. Nonlinear Var. Anal. 3, 189-203 (2019)

23. Bnouhachem, A., Ansari, Q.H., Yao, J.C.: An improvement of alternating direction method for solving variational inequality problems with separable structure. Fixed Point Theory 21(1), 67-78 (2020). https://doi.org/10.24193/fpt-ro.2020.1.05

24. Rockafellar, R.T.: Monotone operators and the proximal point algorithms. SIAM J. Control Optim. 14(5), 877-898 (1976)

25. Takahashi, S., Takahashi, W: Viscosity approximation methods for equilibrium problems and fixed point problems in Hilbert spaces. J. Math. Anal. Appl. 331, 506-515 (2007)

26. Rockafellar, R.T.: On the maximality of sums of nonlinear monotone operators. Trans. Am. Math. Soc. 140, 75-88 (1970)

27. Bruck, R.E., Reich, S.: Nonexpansive projections and resolvents of accretive operators in Banach spaces. Houst. J. Math 3, 459-470 (1977)

28. Li, X.B., Qin, X.L., Rezapour, S., Yao, J.C., Zakeri, S.H.: Hybrid approximate proximal method for vector optimization problems. J. Nonlinear Convex Anal. 20(12), 2471-2494 (2019)

29. Li, X.B., Rezapour, S., Yao, J.C., Zakeri, S.H.: Generalized contractions and hybrid approximate proximal method for vector optimization problems. J. Nonlinear Convex Anal. 21(2), 495-517 (2020)

30. Combettes, P.L., Hirstoaga, S.A.: Equilibrium programming in Hilbert spaces. J. Nonlinear Convex Anal. 6(1), 117-136 (2005)

31. Takahashi, S., Takahashi, W., Toyoda, M.: Strong convergence theorems for maximal monotone operators with nonlinear mappings in Hilbert spaces. J. Optim. Theory Appl. 147, 27-41 (2010)

32. Browder, F.E.: Nonlinear equations of evolution and nonlinear accretive operators in Banach spaces. Bull. Amer. Math. Soc. 73(6), 867-874 (1967) https://projecteuclid.org/euclid.bams/1183529096

33. Xu, H.K.: Viscosity approximation methods for nonexpansive mappings. J. Math. Anal. Appl. 298, $279-291$ (2004)

34. Zegeye, H., Shahzad, N.: Convergence of Mann's type iteration method for generalized asymptotically nonexpansive mappings. Comput. Math. Appl. 62, 4007-4014 (2011)

35. Lin, L.J., Takahashi, W.: A general iterative method for hierarchical variational inequality problems in Hilbert spaces and applications. Positivity 16, 429-453 (2012)

36. Su, Y., Shang, M., Qin, X.: An iterative method of solutions for equilibrium and optimization problems. Nonlinear Anal. 69, 2709-2719(2008) 
37. Takahashi, W., Wong, N.C., Yao, J.C.: Two generalized strong convergence theorems of Halpern's type in Hilbert spaces and applications. Taiwan. J. Math. 16(3), 1151-1172 (2012)

38. liduka, H., Takahashi, W.: Strong convergence theorems for nonexpansive nonself-mappings and inverse-strongly monotone mappings. J. Convex Anal. 11(1), 69-79 (2004)

39. Moudafi, A.: Krasnoselski-Mann iteration for hierarchical fixed-point problems. Inverse Probl. 23, 1635-1640 (2007)

40. Yaoa, Y., Cho, Y.J., Liou, Y.C.: Iterative algorithms for hierarchical fixed points problems and variational inequalities. Math. Comput. Model. 52, 1697-1705 (2010)

41. Peng, J.W., Wang, Y., Shyu, D.S., Yao, J.C.: Common solutions of an iterative scheme for variational inclusions, equilibrium problems and fixed point problems. J. Inequal. Appl. 2008, Article ID 720371 (2008)

Submit your manuscript to a SpringerOpen ${ }^{\circ}$ journal and benefit from:

- Convenient online submission

- Rigorous peer review

Open access: articles freely available online

High visibility within the field

- Retaining the copyright to your article

Submit your next manuscript at $\gg$ springeropen.com 